\title{
On the evolution of aspherical perturbations in the universe: An analytical model
}

\author{
A. Del Popolo ${ }^{1,2,3}$ \\ 1 Dipartimento di Matematica, Università Statale di Bergamo, via dei Caniana 2, 24129 Bergamo, Italy \\ e-mail: adelpop@unibg.it \\ 2 Feza Gürsey Institute, PO box 6 Çengelköy, Istanbul, Turkey \\ 3 Boğaziçi University, Physics Department, 80815 Bebek, Istanbul, Turkey
}

Received 30 October 2001 / Accepted 18 February 2002

\begin{abstract}
I study the role of shear fields on the evolution of density perturbations by using an analytical approximate solution for the equations of motion of homogeneous ellipsoids embedded in a homogeneous background. The equations of motion of a homogeneous ellipsoid (Icke 1973; White \& Silk 1979, hereafter WS) are modified in order to take account of the tidal field, as done in Watanabe (1993) and then are integrated analytically, similar to what was done in WS. The comparison of the analytical solution with numerical simulations shows that it is a good approximation of the numerical one. This solution is used to study the evolution of the configuration of the ellipsoids, to calculate the evolution of the density contrast and that of the axial peculiar velocity of the ellipsoids for several values of the amplitude of the external tidal field, and is compared again with numerical simulations. In order to calculate the evolution of the density contrast at turn-around and collapse velocity at the epoch of collapse, as a function of the ratio of the initial value of the semi-axes, I use the previously-obtained approximate solution to modify the analytical model proposed by Barrow \& Silk (1981) for the ellipsoid evolution in the non-linear regime. The density contrast at turn-around and the collapse velocity are found to be reduced with respect to that found by means of the spherical model. The reduction increases with increasing strength of the external tidal field and with increasing initial asymmetry of the ellipsoids. These last calculations are also compared with numerical solutions and they are again in good agreement with the numerical ones.
\end{abstract}

Key words. cosmology: theory - large-scale structure of Universe - galaxies: formation

\section{Introduction}

Most clusters and superclusters, similar to elliptical galaxies, are not spherical and their shape is not due to rotation (Rood et al. 1972; Gregory \& Tifft 1976; Dressler 1981). This deviation from spherical symmetry is seen wherever large-scale structures can be unambiguously delineated. Going towards very large scales this tendency to the "breaking of symmetry" takes the form of strong inhomogeneity and subclustering. For example, the Local Supercluster (LSC) has an appreciable flatness of the axial ratio, 1:6-1:9 (see Tully 1982). On smaller scales, clusters of galaxies are often highly elongated and galaxies are significantly aspherical. In the framework of the gravitational instability, in which structures formed by the gravitational amplification of small primeval inhomogeneities, this lack of spherical symmetry may be somehow expected. In fact, the perturbations that gave rise to the formation of structures are likely to have been initially aspherical (Barrow \& Silk 1981, hereafter BS; Peacock \& Heavens 1985; Bardeen et al. 1986) and asphericities are then amplified during gravitational collapse (Lin et al. 1965; Icke 1973; BS). The elongations are probably due to a velocity anisotropy of the galaxies (Aarseth \& Binney 1978). According to Binney \& Silk (1979) and to SalvadorSolé \& Solanes (1993) the elongation of clusters originates in the tidal distortion by neighboring protoclusters. In particular, Salvador-Solé \& Solanes (1993) found that the main distortion of a cluster is produced by the nearest neighboring clusters having more than 45 galaxies and the same model can explain the observed alignment between neighboring clusters (Binggeli 1982; Oort 1983; Rhee \& Katgert 1987; Plionis 1994) and that between clusters and their first ranked galaxy (Carter \& Metcalfe 1980; Dressler 1981; Binggeli 1982; Rhee \& Katgert 1987; Tucker \& Peterson 1988; van Kampen \& Rhee 1990; Lambas et al. 1990; West 1994) ${ }^{1}$. As previously reported, another characteristic found by some of the studies previously quoted (e.g., Binggeli 1982), is the tendency that clusters have to align, seen for the first time in the two-dimensional

\footnotetext{
${ }^{1}$ Cluster elongations and alignment could be also explained by means of Zeldovich's (1978) "pancakes" theory of cluster formation but this top-down formation model is probably ruled out for several well-known reasons (Peebles 1993).
} 
distribution of Abell Clusters (Binggeli 1982). The evidence reported suggests that the anisotropy of the external density field plays an important role in the evolution of clusters or superclusters. To be more precise, we mention that the role of shear on structure formation is of fundamental importance. Shear on a density perturbation can be produced by the intrinsic asphericity of the perturbation itself (internal shear) or it can be due to the interaction of the perturbation with the neighboring ones (external shear) ${ }^{2}$. The role of external shear on structure formation was studied theoretically in Binney \& Silk (1979) and Palmer (1983). In particular, Palmer (1983) studied the gravitational collapse of homogeneous prolate spheroids under the influence of a single attractor. The importance of the external shear for structure formation was recognized by Hoffman (1986a, 1989). In particular Hoffman (1986a, 1989), using the quasilinear (QL) approximation (Zel'dovich 1970; Zel'dovich \& Novikov 1983), showed that the shear affects the dynamics of collapsing objects and leads to infall velocities that are larger than in the case of non-shearing ones. As a consequence, according to Hoffmann (1986a, 1989), a general mass element collapses faster than a spherical one. Evrard \& Crone (1992), Bertschinger \& Jain (1994) and Monaco (1995) arrived at similar conclusions. Bertschinger \& Jain (1994) put this result in the form of a theorem according to which spherical perturbations are the slowest in collapsing.

On the accuracy of the Bertschinger \& Jain collapse theorem there is not fully agreement in the literature. For example, according to the previrialization conjecture (Peebles \& Groth 1976; Davis \& Peebles 1977; Peebles 1990), initial asphericities and tidal interactions between neighboring density fluctuations induce significant nonradial motions which oppose the collapse. This kind of conclusion was supported by BS, Szalay \& Silk (1983), Villumsen \& Davis (1986), Bond \& Myers (1993a,b) and Lokas et al. (1996).

In a more recent paper, Audit et al. (1997) have proposed some analytic prescriptions to compute the collapse time along the second and the third principal axes of an ellipsoid, by means of the "fuzzy" threshold approach. They pointed out that the formation of virialized clumps must correspond to the third axis collapse and that the collapse along this axis is slowed down by the effect of the shear rather than being accelerated by it, in contrast to its effect on the first axis collapse. They concluded that spherical collapse is the fastest, in disagreement with Bertschinger \& Jain's theorem.

Van de Weygaert \& Babul (1994) studied the influence of shear fields on the evolution of galactic scale perturbations. They found that the tidal forces induced by large-scale inhomogeneities can affect the mass in and around primordial density peaks and that in some peculiar

\footnotetext{
2 The term "internal shear" has the meaning of "intrinsic asphericity" while "external shear" that of "non-spherical external force".
}

conditions the shear can break up a primordial peak into two distinct halos. Shear is even able to produce the collapse of a void (van de Weygaert 1996).

Internal shear has been studied by means of collapsing homogeneous ellipsoids (Icke 1973; WS; BS). These models show that the evolution of an isolated homogeneous ellipsoid (namely an ellipsoid not exposed to external tidal force) proceeds through a series of uniform ellipsoids, or in other terms the shape of a perturbation does not change much until it acquires a significant overdensity with respect to the background. The collapse time of perturbations of a given initial overdensity, decreases with increasing eccentricity and the collapse is faster for near-oblate configurations than for near prolate ones. BS showed that the density contrast at turn-around and the collapse velocity at pancake formation are reduced relative to the spherical case. Summarizing, the main conclusions are that internal shear can alter the collapse history of structures (Icke 1973; WS). Note that the ellipsoid model has also been used to calculate the cosmological density parameter (WS; BS; Watanabe 1993).

It is important to notice that while the homogeneous ellipsoid model has been widely used in the cosmological context (Palmer 1983; WS; Peebles 1980; BS; Hoffman 1986; Monaco 1995; Eisenstein \& Loeb 1995; Bond \& Myers 1996a,b; van de Weygaert 1996; Audit \& Alimi 1996), only a few papers deal with the study of the effect of shear on the ellipsoid evolution and the analytical studies are even fewer (WS; BS). Moreover, the effect of internal and external shear has been studied separately: for example WS, BS and Watanabe \& Inagaki $(1991,1992)$ neglect the role of tidal forces; Watanabe (1993) studied only the effect of external shear.

It is thus interesting to study the evolution of the homogeneous ellipsoid taking account of both internal and external shear and to look for analytical solutions of the equations describing the evolution of an ellipsoidal perturbation.

In this paper, I shall study the effect of internal and external shear, finding an approximate analytical solution to the equations of motion given in Watanabe (1993). These equations are the equations of Icke (1973) and WS, modified to take account of the effect of the tidal distortion. Similarly to WS, I find an approximate solution to Watanabe's equations which is then compared with numerical simulations.

The paper has two aims:

1) to find an analytical solution for the equations describing the evolution of an ellipsoidal perturbation and to study the changes in configuration, axial velocity fields, and density contrast in terms of the internal shear (intrinsic initial asphericity) and external shear;

2) To study how internal and external shear affect the ellipsoid overdensity at turn-around and axial velocity at the collapse epoch. The final aim is to have some insights in the previrialization conjecture.

I develop this last item by improving the BS model, namely I use the analytical solution of the equations of 
motion, previously found, to calculate the density contrast at turn-around and the axial velocity at the collapse epoch, similar to what is done by BS.

The plan of the paper is the following: in Sect. 2, I introduce the equations of motion of an unisolated ellipsoid. In Sect. 3, I find an approximate analytical solution to the previous equations, similar to what is done by WS for the isolated ellipsoid model. In Sect. 4, I use the previous solution to find the evolution of the density contrast and the axial peculiar velocity. In Sect. 5, the density contrast at turn-around and the velocity at collapse are calculated in order to have some insights on the effects of shear on the collapse of the structure. Section 6 is devoted to conclusions.

\section{Unisolated homogeneous ellipsoid model}

The equations of motion of an irrotational homogeneous ellipsoid with semiaxis lengths $a_{1}, a_{2}$ and $a_{3}$ embedded in a Friedmann-Robertson-Walker background was given by Icke (1973), and WS as:

$\frac{\mathrm{d}^{2} a_{i}}{\mathrm{~d} t^{2}}=-2 \pi G\left[\rho_{\mathrm{e}} \alpha_{i}+\left(\frac{2}{3}-\alpha_{i}\right) \rho_{\mathrm{b}}\right] a_{i}$

where $R_{\mathrm{b}}$ and $\rho_{\mathrm{b}}$ are respectively the scale factor and density of the background universe, $\rho_{\mathrm{e}}$ the density within the ellipsoid. The coefficients $\alpha_{i}$ are given by:

$\alpha_{i}=a_{1} a_{2} a_{3} \int_{0}^{\infty} \frac{\mathrm{d} \lambda}{\left(a_{i}^{2}+\lambda\right)\left[\left(a_{1}^{2}+\lambda\right)\left(a_{2}^{2}+\lambda\right)\left(a_{3}^{2}+\lambda\right)\right]^{\frac{1}{2}}}$.

For the rest of the paper, except Sect. 5, I assume that $a_{1} \leq a_{2} \leq a_{3}$. Note that $\alpha_{\mathrm{i}}$ satisfies $\alpha_{1}+\alpha_{2}+\alpha_{3}=2$, condition coming from the Poisson equation. The equations to solve are then Eq. (1) together with Eq. (2) and the cosmological equation for the Friedmann model:

$\frac{\mathrm{d}^{2} R_{\mathrm{b}}}{\mathrm{d} t^{2}}=-\frac{4 \pi}{3} G \rho_{\mathrm{b}} R_{\mathrm{b}}$

where $R_{\mathrm{b}}$ is the scale factor of the background universe, and the equations of the conservation of mass in each region:

$\rho_{\mathrm{e}} a_{1} a_{2} a_{3}=$ const $\quad \rho_{\mathrm{b}} a_{1} a_{2} a_{3}=$ const.

Equations (1)-(4) describe the evolution of an isolated ellipsoid.

Before going further, let us consider the effects of the over-simplifications of the model:

1) The ellipsoidal approximation, described by the Eqs. (1)-(4), applies mainly in the immediate vicinity of the density extrema (maxima and minima), where the leading terms in the gravitational potential are the quadratic ones. Previous works (see Eisenstein \& Loeb 1995) has shown that the inner region of a perturbation is well approximated by the ellipsoid approximation (Eisenstein \& Loeb 1995 apply this model to a variety of mass scales in the range $10^{8}-10^{15} M_{\odot}$ and WS to superclusters.)
2) The homogeneous isolated ellipsoid model does not take account of the role of the tidal forces due to other nearby objects. This problem can be solved as shown in the rest of this paper or as shown by Eisenstein \& Loeb (1995).

3) The homogeneous ellipsoid model does not take account of the inhomogeneity and of the substructure internal to the system. This last item is a natural limitation of the model: by definition, a homogeneous ellipsoid cannot represent the substructure or inhomogeneity of the object. This limitation, on the one hand, prevents us from treating the distribution of matter and angular momentum within the collapsing object and, on the other hand, has the effect of underestimating the effect of previrialization, and in particular the value of the overdensity at virialization, $\delta_{\mathrm{v}}$ (Peebles 1990). This means that the effect of the shear on the evolution of the density contrast at turnaround and the velocity at collapse, estimated with the ellipsoid model, is even smaller than what one expects in the collapse of a real protostructure.

4) Another limitation of the model is the effect of the matter in the ellipsoid on the smooth background. One expects that the ellipsoid matter causes the density of its immediate surroundings to deviate from the cosmic mean. Then, as a back reaction, tidal fields due to the perturbed external material should induce departure from homogeneity in the ellipsoid. However, when the density inside the ellipsoid, $\rho_{\mathrm{e}}$, is very close to the background density, $\rho_{\mathrm{b}}$, this effect is negligibly small (Watanabe \& Inagaki 1991; WS), while if $\rho_{\mathrm{e}} \gg \rho_{\mathrm{b}}$, the evolution will be determined by its self-gravity (WS). Moreover, numerical simulations have shown that it is a good approximation to ignore departures from homogeneity when one calculates the evolution of the axis ratio (Hui \& Bertschinger 1995).

In spite of the uncertainties listed above, the ellipsoid model gives a good approximation for the evolution of any object that collapses in a fairly homogeneous manner (WS; Eisenstein \& Loeb 1995).

While it is not possible to take account of inhomogeneity, the homogeneous isolated ellipsoid model can be modified in order to take account of external shear as done, for example, by Watanabe (1993). In order to get the quoted goal, one has to get a multipole expansion of the gravitational potential, $\phi$. The gravitational potential at time $t$ and comoving coordinates $(\boldsymbol{x})$, due to the field outside a comoving radius $X$, is given by:

$$
\begin{aligned}
\phi(\boldsymbol{x}, t) & =\sum_{l} \phi_{l}(\boldsymbol{x}, t) \\
& =G \rho_{\mathrm{b}} R_{\mathrm{b}}^{2} \sum_{l} x^{l} \int_{x^{\prime}>X} \frac{\delta\left(\boldsymbol{x}^{\prime}, t\right)}{x^{\prime l+1}} P_{l}\left(\mu^{\prime}\right) \mathrm{d}^{3} x^{\prime}
\end{aligned}
$$

where $P_{l}$ are the Legendre polynomials. In this paper, as I am primarily concerned with shear, I focus on the quadrupole $(l=2)$ terms. In fact, the monopole component, $l=0$, generates no force, since the potential is spatially constant, while the dipole component, $l=1$, induces only streaming motions that cannot alter the shape or induce any rotation. As shown by Eisenstein \& Loeb (1995), 
in the standard CDM scenario, the dipole is generated at large scales, so the object I am studying and its neighborhood move as bulk flow with the consequence that the angular distribution of matter is very small, and then the dipole terms can be ignored. The quadrupole term, $l=2$, is the first term dealing with the effect of tidal distortion:

$\phi_{2}(\boldsymbol{x}, t)=\frac{3}{2} G \rho_{\mathrm{b}} R_{\mathrm{b}}^{2} Q_{i j} x_{i} x_{j}$

where the quadrupole tensor, $Q_{i j}$, is given by:

$Q_{i j}=\int \frac{\delta\left(\boldsymbol{x}^{\prime}, t\right)}{x^{\prime 5}}\left[x_{i}{ }^{\prime} x_{j}{ }^{\prime}-\frac{1}{3} \delta_{i j} \boldsymbol{x}^{\prime 2}\right] \mathrm{d}^{3} x^{\prime}$.

The quadrupole tensor, $Q_{i j}$, is a traceless $3 \times 3$ matrix that can be diagonalized using an appropriate coordinate transformation to get:

$Q_{i j}=Q\left(\begin{array}{ccc}-\beta & 0 & 0 \\ 0 & \beta-1 & 0 \\ 0 & 0 & 1\end{array}\right), \quad 1 / 2 \leq \beta \leq 1$.

In the following, I assume that the field does not vary significantly during the ellipsoid evolution and consequently, for simplicity, the value of $\beta$ is assumed to be constant in time (Watanabe 1993; van de Weygaert 1996). For a positive value of $Q$, which is the component with maximum absolute value in the quadrupole tensor, a perturbation evolves to a prolate configuration and to an oblate one for a negative value of $Q$. If the initial perturbation is spherically symmetric, it shall evolve to an ellipsoid, if all the three components of the quadrupole tensor are different, while, if two components of the quadrupole tensor are equal and $Q>0$ the resulting figure is a prolate spheroid or an oblate one if $Q<0$. If the initial perturbation is an ellipsoid, also in the case that the quadrupole tensor has two equal components, the final configuration is an ellipsoid. In fact, though the cluster becomes a spheroid if the two axes of the external perturbations are equal in Watanabe's (1993) model (because he assumed the initial configuration to be spherical symmetric), generally, the external perturbations are triaxial so the cluster remains an ellipsoid if the initial directions of the axes of the cluster coincides with those of the external perturbations.

The equations of motion are obtained by adding the force due to the potential given by Eq. (6) into Eq. (1). Assuming that the principal axes of the external tidal tensor are always oriented along the principal axes of the mass tensor, the evolution equations reduces to three equations for the three semiaxes of the ellipsoid and are given by (Watanabe 1993; van de Weygaert 1996):

$$
\begin{aligned}
\frac{\mathrm{d}^{2} a_{i}}{\mathrm{~d} t^{2}}= & -2 \pi G\left\{\rho_{\mathrm{e}}\left(\alpha_{i}-\gamma b_{i}\right)+\left[\frac{2}{3}-\left(\alpha_{i}-\gamma b_{i}\right)\right] \rho_{\mathrm{b}}\right\} a_{i} \\
= & -2 \pi G\left[\rho_{\mathrm{e}} \alpha_{i}+\left(\frac{2}{3}-\alpha_{i}\right) \rho_{\mathrm{b}}\right] a_{i} \\
& -2 \pi G \gamma\left(-b_{i}\right)\left(\rho_{\mathrm{e}}-\rho_{\mathrm{b}}\right) a_{i}
\end{aligned}
$$

where:

$\gamma=\frac{3}{2 \pi} \frac{Q}{\delta}, \quad \boldsymbol{b}=(-\beta, \beta-1,1)$
Note that in the rightmost term of Eq. (9), I wrote $-b_{i}$ to display the equation in the same form as van de Weygaert (1996), in order to simplify the comparison with the result of that paper. A comparison of Eq. (1) with Eq. (9) shows that the evolution of the ellipsoid is modified by the tidal force if the term $\gamma b_{i}$ is large, while the evolution of the ellipsoid is dominated by self-gravity if this term is small. I implicitly assume that the external structures, giving rise to the tidal field, are at a large distance from the ellipsoid (see Eisenstein \& Loeb 1995). As a consequence, the amplitude of the external quadrupole force is assumed to increase with the linear growth rate (Ryden 1988; Watanabe 1993; Eisenstein \& Loeb 1995), $D(t)$ (this last quantity is given in Peebles 1980):

$Q(t)=Q_{0} \frac{D(t)}{D_{0}}$

Here the subscript " 0 " means that the corresponding quantity is calculated at the present epoch and $D(t)=$ $R_{\mathrm{b}}(t)$, for an Einstein-de Sitter (hereafter EdS) universe. Similar to WS, I assume that initially $R_{\mathrm{b}}=1$. In the particular case of an isolated ellipsoid with $a_{1}: a_{2}: a_{3}=$ $1: 1.25: 1.5, R_{\mathrm{b}}=873$ at collapse. For sake of precision, I want to stress that in an Einstein-de Sitter universe, Eq. (11), $Q(t)=Q_{0} \frac{R_{\mathrm{b}}(t)\left(t / t_{0}\right)^{2 / 3}}{R_{\mathrm{b}}\left(t_{0}\right)}$ reduces to $Q\left(t_{0}\right)=Q_{0}$ at time $t_{0}$.

In order to have an estimate of the value of $Q_{0}$, for a cluster interacting with a neighboring one, I use the simple model in Watanabe (1993). I consider a cluster which has a neighboring cluster with a mean density contrast $\langle\delta\rangle \simeq$ 3 , a comoving separation $\left(0,0, x_{3}\right)$, and a comoving size $\Delta x_{3}=x_{3} / 3$. The $Q_{33}$ quadrupole component is given by:

$Q_{33} \simeq \frac{8}{9} \pi<\delta>\left(\frac{\Delta x_{3}}{x_{3}}\right)^{3} \simeq 0.3$.

The previous estimate corresponds to a cluster interacting with a neighbor having a mass excess comparable to that of the Virgo cluster, and a separation three times its size.

Another way of estimating $Q_{0}$ is by using the anisotropy of the velocity field in the LSC from data of Lilje et al. (1986). If we indicate with $Q_{\mathrm{v} 0}$ the component of the largest absolute value of the anisotropic velocity, one gets: $Q_{0} \Omega_{0}^{0.6}=\frac{4 \pi}{3} Q_{\mathrm{v} 0}$ (Watanabe 1993). Since Lilje et al. (1986) deduced a value of $Q_{\mathrm{v} 0} \sim 0.1-0.2$ at the distance of the Local Group from Virgo, we have that $Q_{0} \Omega_{0}^{0.6} \sim 0.4-0.8$

Before going on, it is important to discuss a basic difference between the present paper and that of Watanabe (1993). Unlike from Watanabe (1993), in this paper I assume that protostructures have an initial asphericity, while the paper of Watanabe (1993) (similarly to those of van de Weigaert 1996 and Palmer 1983) assume that the initial configuration is spherical, so that the principal axes of the external tidal tensor will be oriented along the principal axes of the mass tensor and the equations of motions reduce to three equations involving the diagonal components of the traceless tidal tensor. Our assumption 
of initial asphericity of protostructures is motivated by the fact that previous analyses of the topology of the constant-density profiles in the neighborhood of the peaks of the Gaussian field showed that the isodensity surfaces are simply connected and approximately ellipsoidal (Doroshkevich 1970; Bardeen 1986). We also know that the initial asphericity has a certain role in shaping the final configuration of the structure (Icke 1973; WS; BS). By means of this assumption, we have the noteworthy advantage of studying the joint effect of "internal and external shear" (see the final part of Sect. 3 for a discussion). The approach of this paper, assuming that the principal axes of the external tidal tensor are always oriented along the principal axes of the mass tensor, is chosen for mathematical simplicity. Note that, at the same time, the assumption is not strange or without motivation: for example van de Weygaert \& Babul (1994), in order to study the effect of shear fields on the evolution of galactic scale density peaks, make a similar assumption, namely that the shear tensor, at the location of the peak representing the structure, is oriented so that it is diagonal. Moreover, in a recent paper Porciani et al. (2002) find a strong alignement between the principal axes of the inertia and the shear tensor, in contradiction to usual assumption that the two tensors are largely uncorrelated (Hoffman 1986b; Heavens \& Peacock 1988; Catelan \& Theuns 1996).

Finally, I want to stress that the approximate solution found in the present paper give a more general representation of structure formation than those described, as examples, in the following sections. For example, the assumptions and results of Watanabe (1993) are re-obtained assuming that the three axes of the ellipsoid are equal, or in other words Watanabe's result is a "particular case" of those of this paper when $a_{1}\left(t_{i}\right)=a_{2}\left(t_{i}\right)=a_{3}\left(t_{i}\right)$. Moreover, in the present paper, I derive analytical solutions for the equations of motion of the ellipsoid, while Watanabe (1993) solve the same equations numerically.

\section{An analytical approximate solution}

An analytical approximation of the solution describing the evolution of the $i$ th axis of the isolated ellipsoid was found by WS. The equations of motion can be integrated analytically if one assumes that:

1) The evolution of the configuration is self-similar, which means that $\alpha_{i}(t) \simeq \alpha_{i}\left(t_{i}\right)$.

2) The time dependences of $\rho_{\mathrm{e}} a_{i}$ and $\rho_{\mathrm{b}} a_{i}$ are the same as in the spherical model, namely:

$\left(\rho_{\mathrm{e}} a_{i}\right)(t)=\frac{-3}{4 \pi G} \ddot{R}_{\mathrm{s}} \frac{R_{\mathrm{b}}\left(t_{i}\right)}{R_{\mathrm{s}}\left(t_{i}\right)}=\frac{-3}{4 \pi G} \ddot{R}_{\mathrm{e}} \frac{R_{\mathrm{b}}\left(t_{i}\right)}{R_{\mathrm{e}}\left(t_{\mathrm{i}}\right)}$

where $R_{\mathrm{S}}$ is the radius of a spherical shell whose initial density enhancement within $a_{\mathrm{s}}$ is $\delta_{\mathrm{s}}\left(t_{i}\right)=\delta_{\mathrm{e}}\left(t_{i}\right)$ and

$\left(\rho_{\mathrm{b}} a_{i}\right)(t)=\frac{-3}{4 \pi G} \ddot{R}_{\mathrm{b}} \frac{a_{i}\left(t_{i}\right)}{R_{\mathrm{b}}\left(t_{i}\right)}$.
Defining $R_{\mathrm{s}}\left(t_{i}\right)=R_{\mathrm{b}}\left(t_{i}\right)=1$, and substituting Eqs. (13), (14) into Eq. (1) and integrating, one obtains:

$\frac{a_{i}(t)}{a_{i}\left(t_{i}\right)}=R_{\mathrm{b}}-\frac{3}{2} \alpha_{i}\left(R_{\mathrm{b}}-R_{\mathrm{e}}\right)$

(WS). This approximation gives a good representation for the evolution of the semiaxes of the ellipsoid for configurations not too extreme. A comparison between the numerical solution of the equations of motion, obtained using a Bulirsch-Stoer scheme, and the prediction of Eq. (15) is shown in Figs. 1a-c. In all the cases studied, the ellipsoids are embedded in an EdS background universe with Hubble parameter $H_{0}=50 \mathrm{~km} \mathrm{~s}^{-1} / \mathrm{Mpc}$ and $\rho_{\mathrm{e}} / \rho_{\mathrm{b}}=1.003$. The velocity perturbation is taken to correspond to the growing mode solution of the linear perturbation theory. Calculations are terminated when the shortest axis becomes zero. In Figs. 1a-c, the ellipsoids have initial axial ratios of $a_{1}: a_{2}: a_{3}=1: 1.25: 1.5,1: 1.5: 3,1: 1.5: 5$, respectively. The solid lines represent the result of the numerical solutions and the dotted lines the approximate analytical solution (Eq. (15)). The top line represent the longest axis evolution and the bottom one that of the shortest one. In this section, I am principally interested in testing the goodness of the approximation and less in describing the general properties of the evolution of the perturbation, a point which was widely discussed in WS and several other papers. However, two interesting points that emerge from the calculation are worth noting:

a) the shape of a perturbation is conserved until it acquires a significant overdensity with respect to the background. b) The collapse time of perturbations of fixed initial overdensity, for a fixed background, decreases with increasing initial asymmetry. In other words the internal shear influences the collapsing region geometry. More anisotropic initial configurations are characterized by an acceleration of the collapse along the shortest axis and a slowing down along the longest one. As we shall see later, a similar effect is produced by the external shear.

Coming back to the goodness of the approximation of Eq. (15) we have, for example, in the first case $\left(a_{1}: a_{2}: a_{3}=\right.$ $1: 1.25: 1.5)$, that the error in $a_{2}\left(t_{\mathrm{c}}\right)$ is $\simeq 7 \%$ while that in $a_{3}\left(t_{\mathrm{c}}\right)$ is $\simeq 8 \%$, where $t_{\mathrm{c}}$ is the collapse time of the first axis. For a configuration $a_{1}: a_{2}: a_{3}=1: 1.5: 3$, Fig. $1 \mathrm{~b}$ shows that Eq. (15) gives a worse approximation going from less asymmetric to more asymmetric configurations, especially in the case of the shortest axis. This trend is confirmed by Fig. 1c, representing the initial configuration $a_{1}: a_{2}: a_{3}=1: 1.5: 5$. The same problem was encountered by Watanabe \& Inagaki (1991) in the calculation of the axial peculiar velocity using the WS approximate solution. It is possible to improve the approximation modifying Eq. (15) slightly, and introducing some parameters whose values can be obtained using the least-square method:

$$
\begin{aligned}
& \frac{a_{1}(t)}{a_{1}\left(t_{i}\right)}=R_{\mathrm{b}}-\frac{3}{2} \alpha_{1}\left(R_{\mathrm{b}}-R_{\mathrm{e}}\right)-d \times R_{\mathrm{b}}^{\left(\frac{2+3 c_{1}}{2}\right)}\left(1-\frac{3 \alpha_{1}}{2}\right) \\
& \frac{a_{2}(t)}{a_{2}\left(t_{i}\right)}=R_{\mathrm{b}}-\frac{3}{2} c_{2} \alpha_{2}\left(R_{\mathrm{b}}-R_{\mathrm{e}}\right)
\end{aligned}
$$




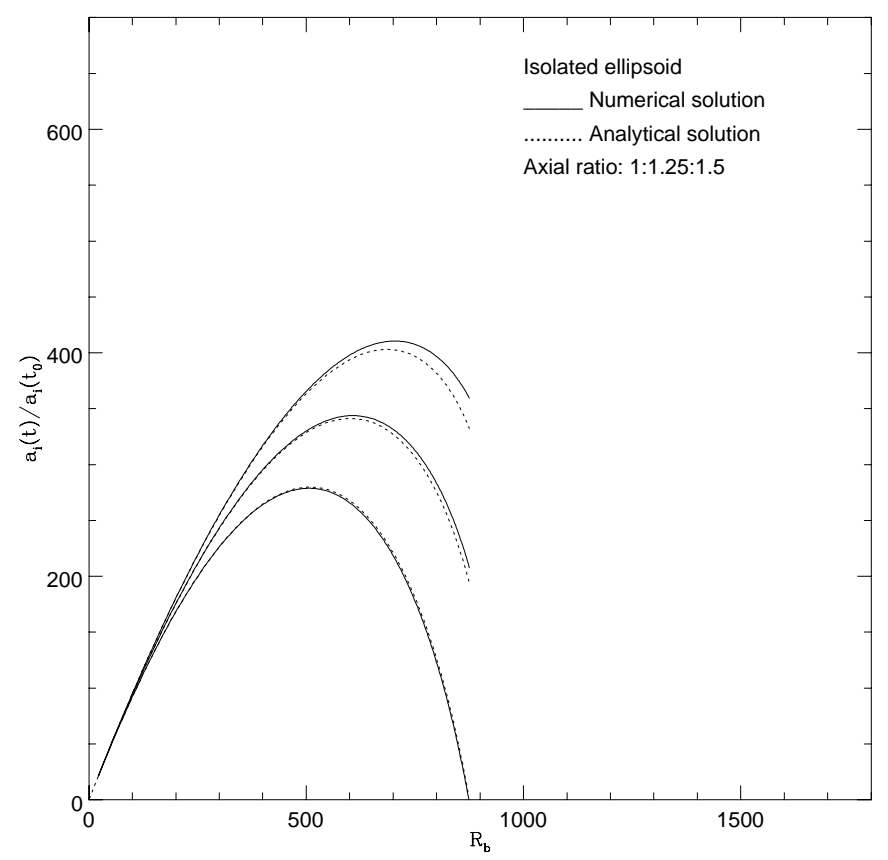

(a)

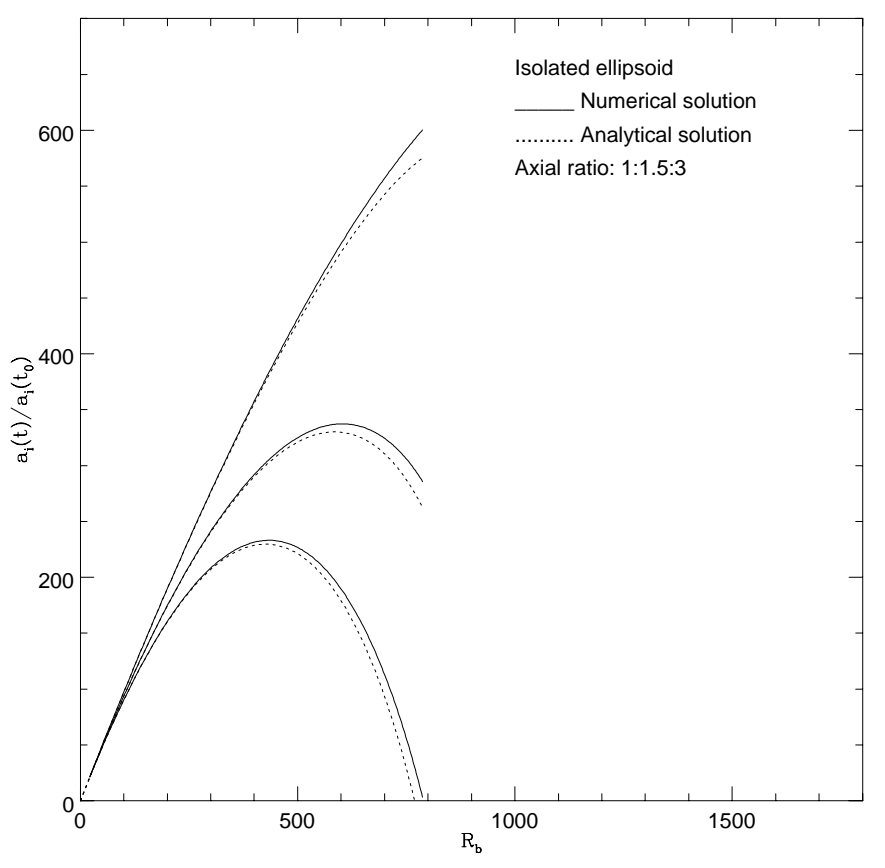

(b)

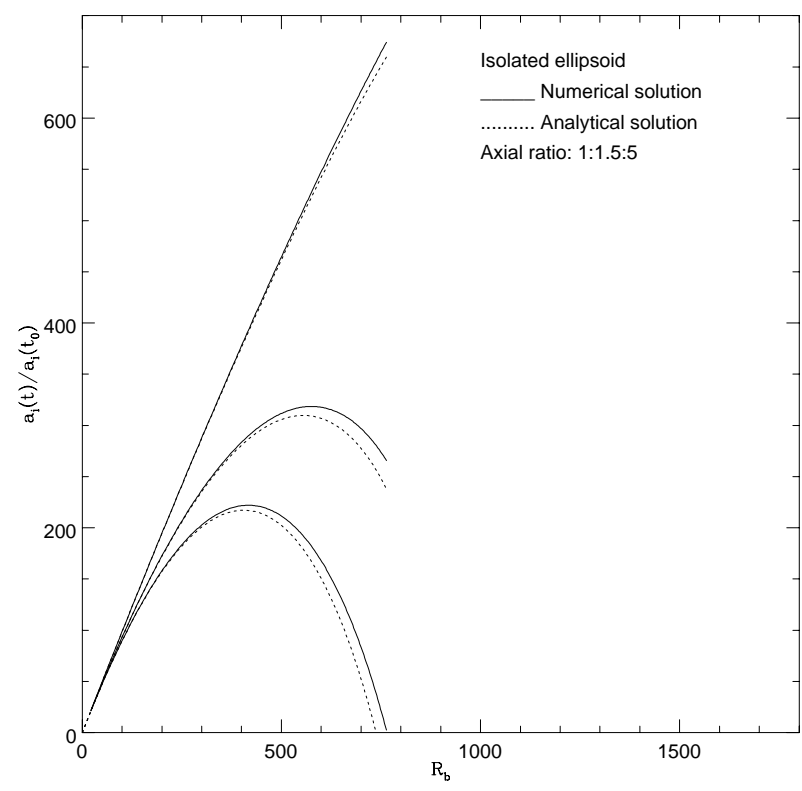

(c)

Fig. 1. a)-c) Evolution of isolated homogeneous ellipsoidal perturbations in an EdS universe with $H_{0}=50 \mathrm{~km} \mathrm{~s} / \mathrm{Mpc}$, $\rho_{\mathrm{e}} / \rho_{\mathrm{b}}=1.003$ and axial ratio 1:1.25:1.5 a), 1:1.5:3 b), 1:1.5:5 c). The solid lines represent numerical solutions obtained using Bulirsh-Stoer algorithm while the dotted ones the approximate analytical solution of WS.

$\frac{a_{3}(t)}{a_{3}\left(t_{i}\right)}=R_{\mathrm{b}}-\frac{3}{2} c_{3} \alpha_{3}\left(R_{\mathrm{b}}-R_{\mathrm{e}}\right)$

For ellipsoids having an initial axial ratio $1: a_{2}: a_{3}$ with $a_{1} \geq 1.25$ and $a_{2} \geq 1.5$, we have that $d=7.22 \times 10^{-7}$ and $c_{i}$ is given by:

$c_{1}=a_{10}^{-0.115} a_{20}^{0.035} a_{30}^{0.08}, \quad c_{2}=a_{10}^{0.07} a_{20}^{-0.06} a_{30}^{-0.01}$, $c_{3}=1.002 a_{10}^{0.1} a_{20}^{-0.035} a_{30}^{-0.065}$ where $a_{i 0}$ represents the initial value of the $i$ th axis.

Figures $1 \mathrm{~d}-\mathrm{f}$ shows that Eqs. (16)-(18) give a better representation of numerical results, with respect to Eq. (15), for all of the three axes (the initial axial ratio is the same as Figs. $1 \mathrm{a}-\mathrm{c})$.

Similarly to the case of an isolated ellipsoid, it is possible to obtain an analytical solution of Eq. (9), describing 


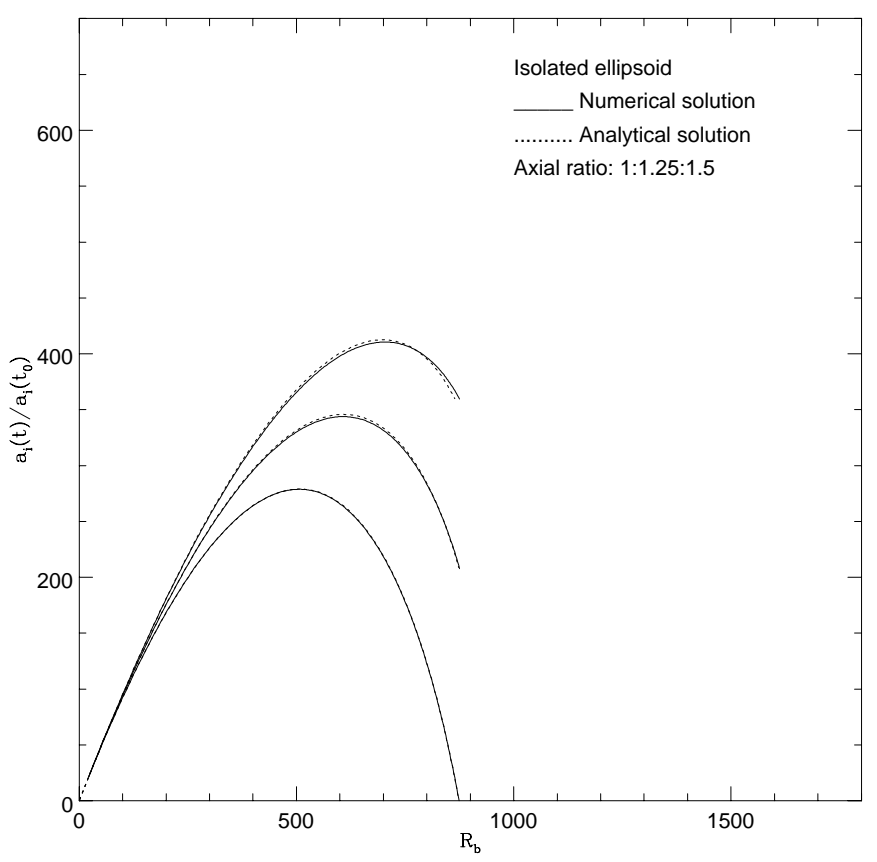

(d)

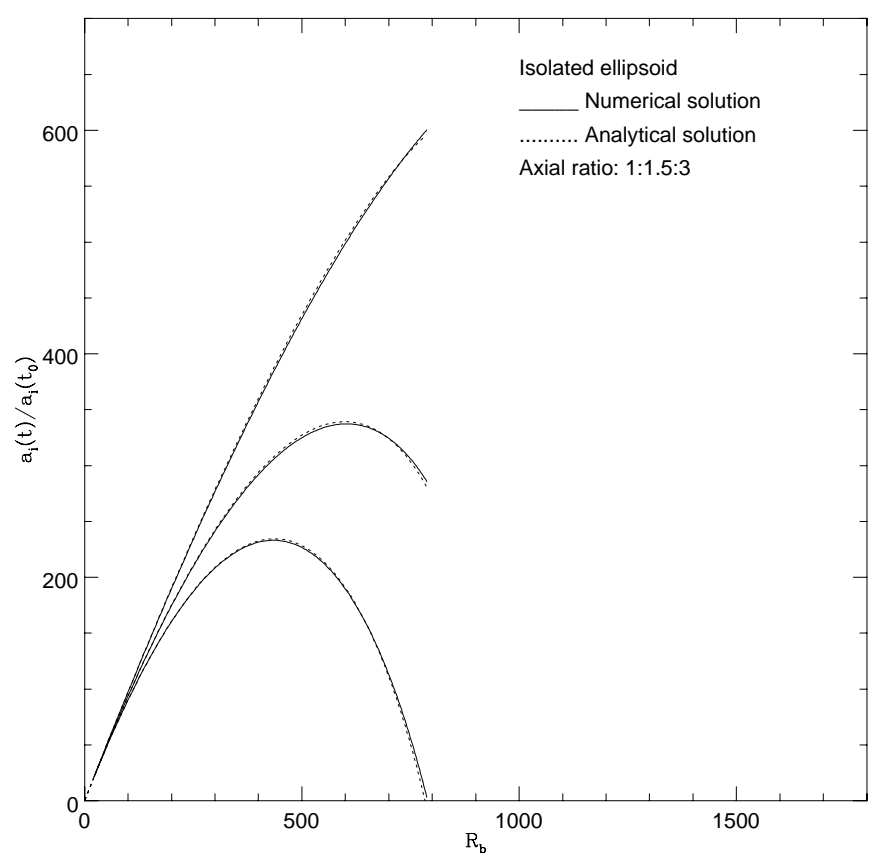

(e)

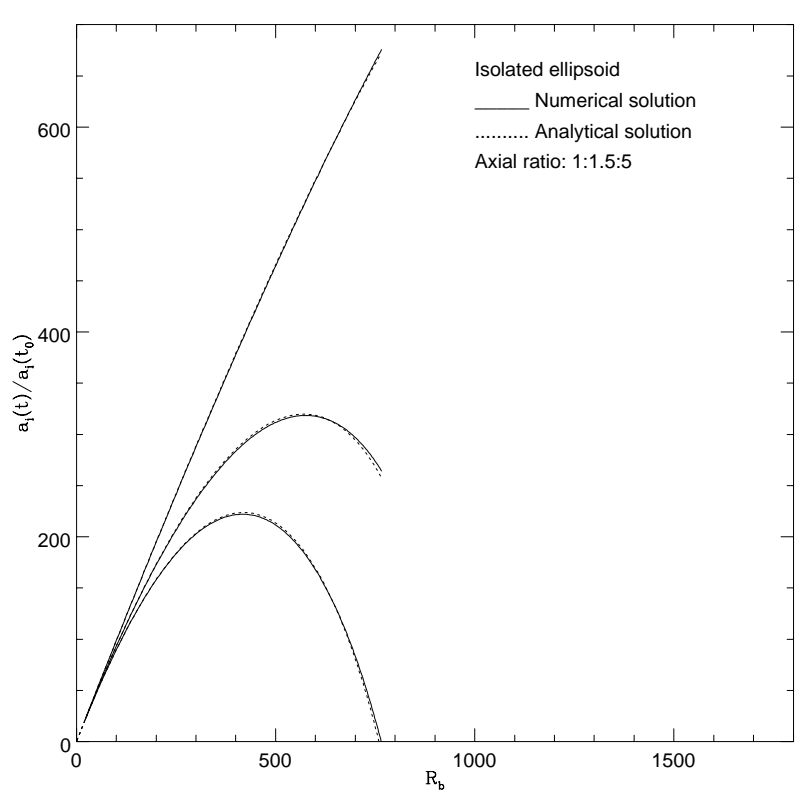

(f)

Fig. 1. d)-f) Same as the previous figures Figs. 1a-c but now the WS analytical approximate solution is improved by using some free parameters fitted to the numerical solution by means of the least-square method (see text for a description).

the evolution of an unisolated ellipsoid. In this case the solution can be written in the form:

$\frac{a_{1}(t)}{a_{1}\left(t_{i}\right)}=R_{\mathrm{b}}-\frac{3}{2} \tilde{\alpha}_{1}\left(R_{\mathrm{b}}-R_{\mathrm{e}}\right)-d \times R_{\mathrm{b}}^{\left(\frac{2+3 c_{1}}{2}\right)}\left(1-\frac{3 \tilde{\alpha}_{1}}{2}\right)$

$\frac{a_{2}(t)}{a_{2}\left(t_{i}\right)}=R_{\mathrm{b}}-\frac{3}{2} \tilde{\alpha}_{2}\left(R_{\mathrm{b}}-R_{\mathrm{e}}\right)$
$\frac{a_{3}(t)}{a_{3}\left(t_{i}\right)}=R_{\mathrm{b}}-\frac{3}{2} \tilde{\alpha}_{3}\left(R_{\mathrm{b}}-R_{\mathrm{e}}\right)$

For ellipsoids having the initial axial ratio $1: a_{2}: a_{3}$, with $a_{1} \geq 1.25$ and $a_{2} \geq 1.5$, we now have that $c_{1}=1.23$, $d=6 \times 10^{-7}$ and:

(21) $\quad \tilde{\alpha}_{1}=\alpha_{1}+0.0672\left(\frac{b_{1}}{b_{2}}\right)^{0.15} b_{3}^{0.6}$ 
$\tilde{\alpha}_{2}=a_{10}^{0.07} a_{20}^{-0.06} a_{30}^{-0.01}\left[\alpha_{2}+0.031\left(\frac{b_{2}}{b_{1}}\right)^{0.5} b_{3}^{0.95}\right]$

$\tilde{\alpha}_{3}=1.002 a_{10}^{0.1} a_{20}^{-0.035} a_{30}^{-0.065}\left(\alpha_{3}-0.063 a_{30}^{0.09} b_{3}^{0.95}\right)$

where $b_{i}$ was defined in Eq. (10).

In the case of prolate spheroids, with axial ratio 1:1: $a_{3}$ and $1 \leq a_{3} \leq 5$, a better approximation to the $\alpha_{i}$ is:

$\tilde{\alpha}_{1}=\alpha_{1}+0.037\left(\frac{a_{30}}{a_{10}}\right)^{0.35}\left(\frac{b_{1}}{b_{2}}\right)^{0.15} b_{3}^{0.6}$

$\tilde{\alpha}_{2}=\left(\frac{a_{10}}{a_{30}}\right)^{0.01}\left[\alpha_{2}+0.031\left(\frac{a_{10}}{a_{30}}\right)\left(\frac{b_{2}}{b_{1}}\right)^{0.5} b_{3}^{0.95}\right]$

$\tilde{\alpha}_{3}=1.002\left(\frac{a_{20}}{a_{30}}\right)^{0.065}\left(\alpha_{3}-0.063 a_{30}^{0.09} b_{3}^{0.95}\right)$.

The comparison between the numerical results and Eqs. (20)-(22) are shown in Figs. 2a-c (the notation in Figs. 2a-2c is the same as Figs. 1a-f). In Figs. 2a-c, I study the evolution of an ellipsoid having initial axes in the ratio 1:1.25:1.5 for different values of $Q_{0}$, and $b_{i}$ $\left(b_{i}=(-0.5,-0.5,1)\right.$, and $\left.Q_{0}=0.1,0.2,0.4\right)$. These figures show that for a fixed initial configuration the effect of the external tidal field causes a qualitatively different evolution with respect to an isolated ellipsoid. The ellipsoid expands less rapidly along the $x$ and $y$ axes, due to the extra decelerating effect of the positive tidal components $\left(-b_{i}=(0.5,0.5,-1)\right)$, and more rapidly along the $z$ axis, since the tidal force is negative along this axis, and this effect increases with increasing strength of the external tidal field. Summarizing, the effect of a positive tidal force along a given axis is to slow down the expansion of the ellipsoid along this axis, and the effect of a negative tidal field is to accelerate the expansion of the ellipsoid along that axis. In other words, shear produce dilation in one direction and contraction in the other two and vice versa (contraction in one direction and dilation in the other two), according to its configuration. The result is in agreement with van de Weygaert (1996) and Audit et al. (1997) (note that Eq. (9) and Eq. (1) of van de Weygaert (1996) differ for the sign of the tidal tensor components, such that the evolution of the axes results inverted with respect to our model). One may expect that the shear can change the nature of the secondary infall, influencing the final mass acquired by a peak and the density profiles of halos (Bond \& Myers 1993; van de Weygaert \& Babul 1994). This idea is confirmed by Figs. $2 \mathrm{a}-\mathrm{c}$, which show that the shear produces a decrease in the collapse time of perturbations of fixed initial overdensity, for a fixed background, and that the effect increases with increasing strength of the external field. It is natural to expect that, for some shear configurations and a strong enough external field, even the core region of a perturbation can be induced to fragment, giving rise to different halos (van de Weygaert \& Babul 1994), or, as shown by van de Weygaert (1996) and
Audit et al. (1997), the external tidal field can induce even a void to collapse, if the external field is strong enough.

In Figs. 2d-e, I plot the evolution of the ellipsoids for a fixed value of $Q_{0}$ and $b_{i}$ and for different values of the initial axial ratio, in order to study the effect of the internal shear. In Figs. 2d-e, the axial ratio is 1:1.25:2, 1:1.25:5, respectively, $Q_{0}=0.1$ and $b_{i}=(-0.5,-0.5,1)$. The plots show a situation similar to that seen in the case of external shear: namely, the collapse time of the perturbation decreases with increasing initial anisotropy. While for isolated ellipsoids or for unisolated ones with small values of $|\gamma|$, the axial ratio does not appreciably change until the perturbation enters a strongly nonlinear regime (the self-gravity is dominant); for large values of $|\gamma|$, the collapse is anisotropic even for small values of $\delta$. This means that the collapsing region geometry is strongly influenced by the external shear. If the external field is strong enough, the external shear is dominant in shaping the region with respect to internal shear, in agreement with Eisenstein \& Loeb (1995) and Watanabe (1993).

As discussed in Sect. 2, in this paper, unlike Watanabe (1993), I assume that protostructures have an initial asphericity. By means of this assumption, I have the noteworthy advantage of being able to study the joint effect of "internal and external shear". So it is interesting to analyse the contribution to the asphericity of real clusters of galaxies (e.g. LSC) coming from inner shear and external shear. In the case of an isolated ellipsoid the length of the uncollapsed axes at collapse can be obtained similarly to WS, by means of Eqs. (17), (18):

$\frac{a_{3}\left(t_{\mathrm{c}}\right)}{a_{2}\left(t_{\mathrm{c}}\right)}=\frac{a_{3}\left(t_{i}\right)}{a_{2}\left(t_{i}\right)} \frac{R_{\mathrm{b}}-\frac{3}{2} c_{3} \alpha_{3}\left(R_{\mathrm{b}}-R_{\mathrm{e}}\right)}{R_{\mathrm{b}}-\frac{3}{2} c_{2} \alpha_{2}\left(R_{\mathrm{b}}-R_{\mathrm{e}}\right)}$

where $t_{\mathrm{c}}$ is obtained by $a_{1}\left(t_{\mathrm{c}}\right)=0$. Notice that the approximation given in WS (Eqs. (11) or (13)) is not very accurate for extreme configurations.

In the case of an unisolated ellipsoid the length of the uncollapsed axes at collapse can be obtained by means of Eqs. (21) $-(22)$ :

$\frac{a_{3}\left(t_{\mathrm{c}}\right)}{a_{2}\left(t_{\mathrm{c}}\right)}=\frac{a_{3}\left(t_{i}\right)}{a_{2}\left(t_{i}\right)} \frac{R_{\mathrm{b}}-\frac{3}{2} \tilde{\alpha}_{3}\left(R_{\mathrm{b}}-R_{\mathrm{e}}\right)}{R_{\mathrm{b}}-\frac{3}{2} \tilde{\alpha}_{2}\left(R_{\mathrm{b}}-R_{\mathrm{e}}\right)}$

If we consider an initial isolated ellipsoid with $a_{1}: a_{2}: a_{3}=$ $1: 1.25: 1.5$, I get at collapse a ratio $\frac{a_{3}\left(t_{c}\right)}{a_{2}\left(t_{c}\right)} \simeq 1.9$. For the same initial axial ratio and $b=(-0.5,-0.5,1), Q_{0}=0.1$, I get $\frac{a_{3}\left(t_{c}\right)}{a_{2}\left(t_{c}\right)} \simeq 2.5$ which represents an increase of $\simeq 34 \%$ of the previous ratio. Increasing the value of $Q_{0}$ to 0.2 implies that $\frac{a_{3}\left(t_{\mathrm{c}}\right)}{a_{2}\left(t_{\mathrm{c}}\right)} \simeq 3.3$ and so an increase of $\simeq 76 \%$ of the previous ratio. If $Q_{0}=0.4$, I get $\frac{a_{3}\left(t_{c}\right)}{a_{2}\left(t_{c}\right)} \simeq 5.9$, indicating a dominant role of external shear in shaping structures for high $Q_{0}$. We can study the effect of varying the initial configuration of ellipsoids in the presence of external shear. In the case of an isolated ellipsoid with $a_{1}: a_{2}: a_{3}=1: 1.25: 1.7$, I get $\frac{a_{3}\left(t_{c}\right)}{a_{2}\left(t_{c}\right)} \simeq 2.8$. If the ellipsoid is unisolated $\left(b=(-0.5,-0.5,1), Q_{0}=0.1\right)$, I get $\frac{a_{3}\left(t_{\mathrm{c}}\right)}{a_{2}\left(t_{\mathrm{c}}\right)} \simeq 3.7$. 


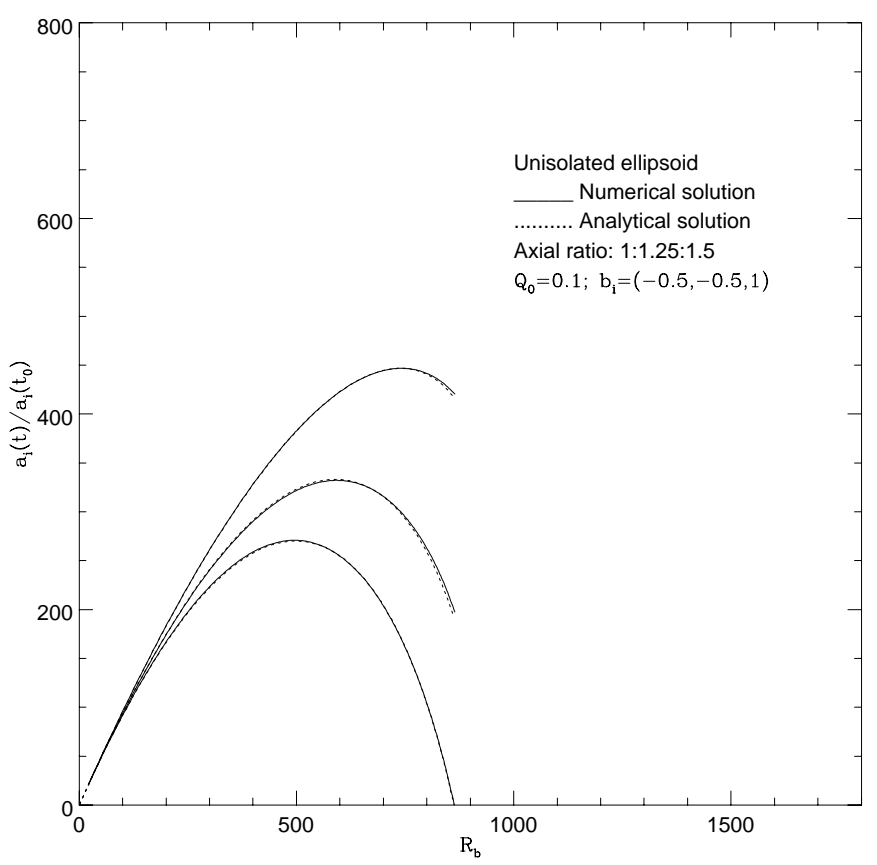

(a)

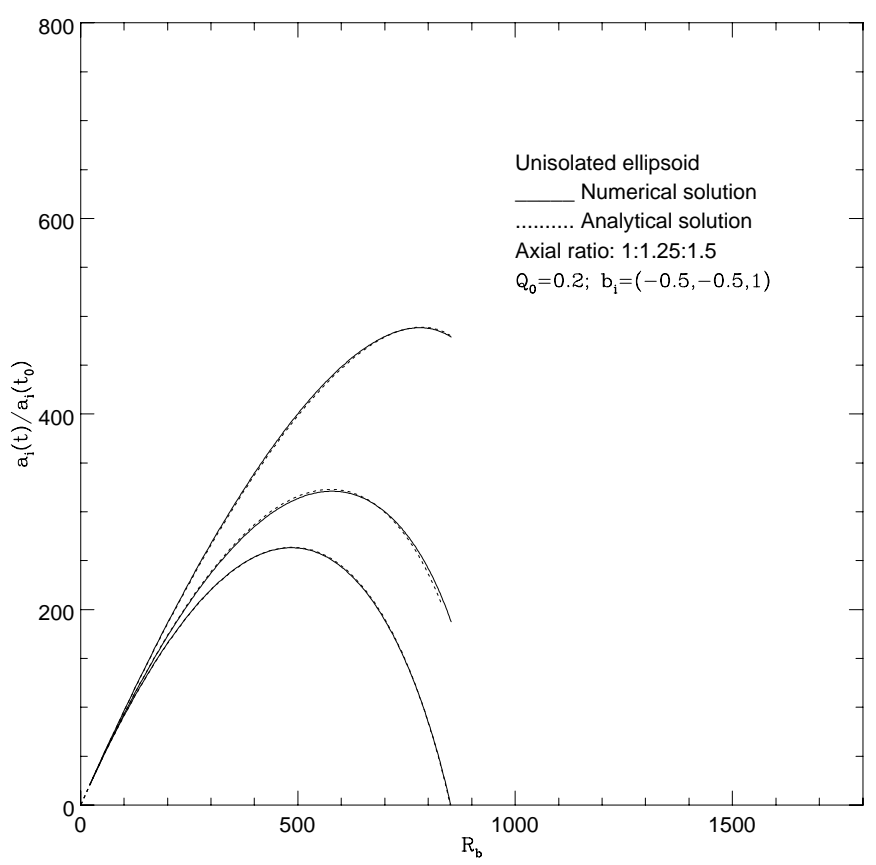

(b)

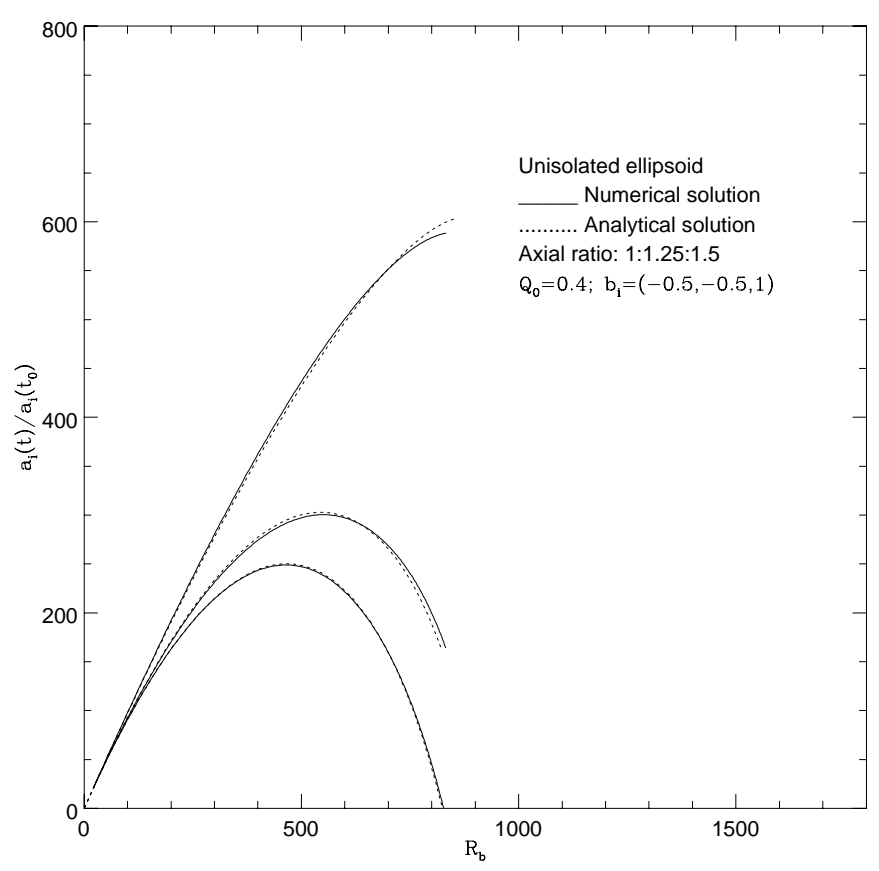

(c)

Fig. 2. a)-c) Evolution of unisolated homogeneous ellipsoidal perturbations in an EdS universe with $H_{0}=50 \mathrm{kms} \mathrm{s}^{-1} / \mathrm{Mpc}$, $\rho_{\mathrm{e}} / \rho_{\mathrm{b}}=1.003$, axial ratio is $1: 1.25: 1.5, b_{i}=(-0.5,-0.5,1)$, and $\left.\left.\left.Q_{0}=0.1 \mathbf{a}\right), Q_{0}=0.2 \mathbf{b}\right), Q_{0}=0.4 \mathbf{c}\right)$.

The isolated ellipsoid with $a_{1}: a_{2}: a_{3}=1: 1.25: 2$ gives $\frac{a_{3}\left(t_{c}\right)}{a_{2}\left(t_{c}\right)} \simeq 4.6$ while the unisolated $(b=(-0.5,-0.5,1)$, $Q_{0}=0.1$ ) one gives $\frac{a_{3}\left(t_{c}\right)}{a_{2}\left(t_{c}\right)} \simeq 6$.

The previous are only some examples (summarized in Table 1) of how the model can be used to obtain information on the effect of external and internal shear on structure formation.

\section{Evolution of the axial peculiar velocity and of the density contrast}

The approximate solution obtained in the previous section can be used to calculate the evolution of the axial peculiar velocity. I use Eqs. (20)-(22) to calculate the peculiar 


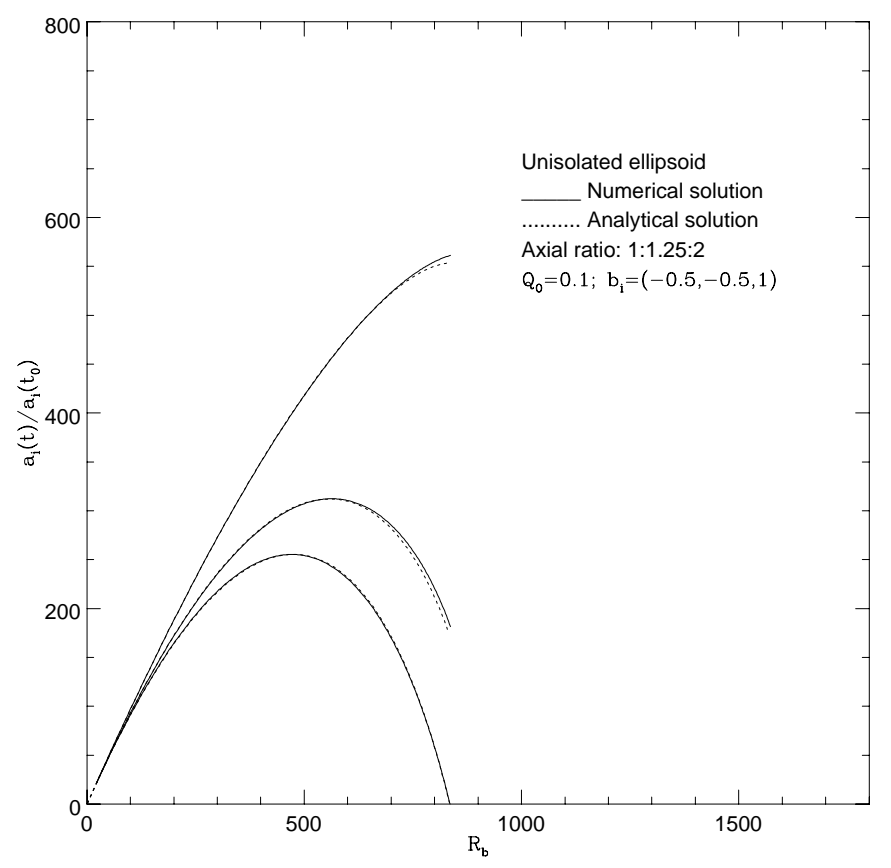

(d)

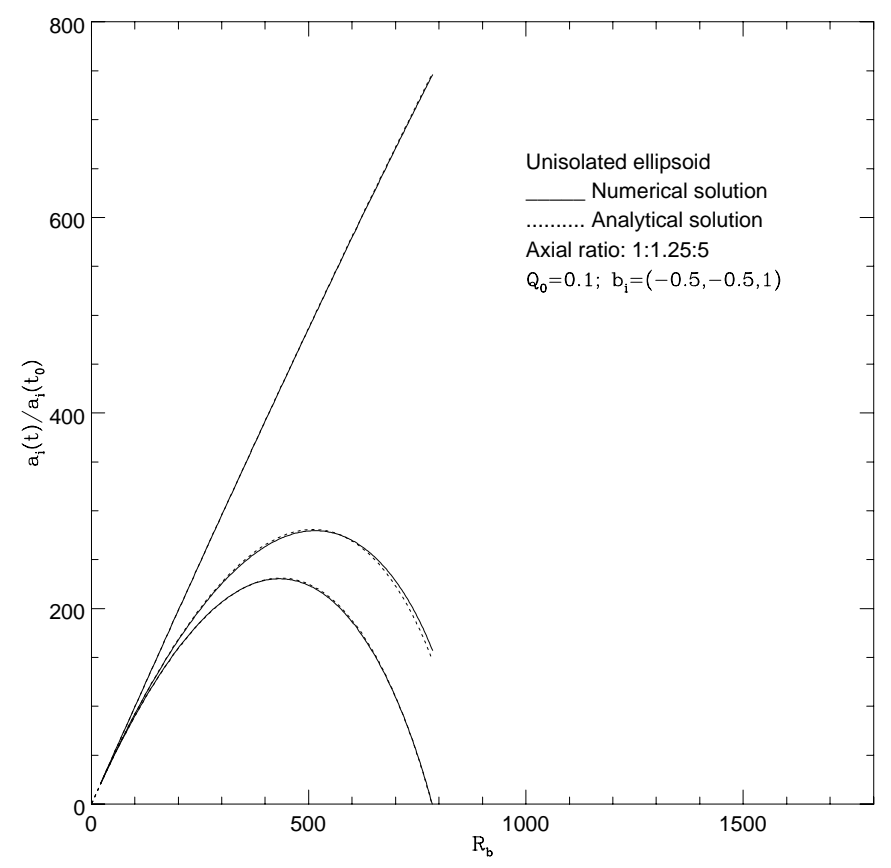

(e)

Fig. 2. d)-e) Same as the previous Fig. 2a but now the axial ratio is 1:1.25:2 d), 1:1.25:5 e), while $Q_{0}=0.1$ and $b_{i}=$ $(-0.5,-0.5,1)$.

velocity along the axis of the ellipsoid in units of the Hubble expansion velocity, $H$ :

$$
\frac{v_{\mathrm{i}}}{H a_{i}}=\left|\frac{\dot{a}_{i}}{H a_{i}}-1\right|
$$

The results of the calculation are plotted in Figs. 3a-e. In Figs. 3a-c, I study how the internal shear influences the evolution of the velocity. In all three figures, the solid line represents, from top to bottom, the numerical result for the peculiar velocity along the shortest, medium and longest axis. The dotted line represents the velocity obtained from Eq. (31) using the approximation for the semiaxes (Eqs. (20)-(22)). Going from Figs. 3a to 3c, the value of $Q_{0}$ is $0.1, b_{i}=(-0.5,-0.5,1)$ and the axial ratio $1: 1.25: 1.5,1: 1.25: 5,1: 2: 3$, respectively. For the goodness of the approximation, the plots show that the approximate solution is in good agreement with the numerical results. From a physical point of view, the plots show that there is a considerable difference between the peculiar velocities of the longest and shortest axes and in particular that the shorter the axis, the larger the velocity of collapse.

The effect of the external field is shown in Figs. 3d-e. The solid line in Fig. 3d represents the peculiar velocity for an ellipsoid of axial ratio 1:1.25:1.5 when no external field is present, while the dotted and dashed lines represent the case $Q_{0}=0.1, b_{i}=(-0.5,-0.5,1)$, and $Q_{0}=0.2, b_{i}=(-0.5,-0.5,1)$, respectively. The situation is similar to that seen when I described the evolution of the semiaxes for different values of the external field. External shear produces different effects on the axes: the evolution of the longest axis, which is characterized by the smallest

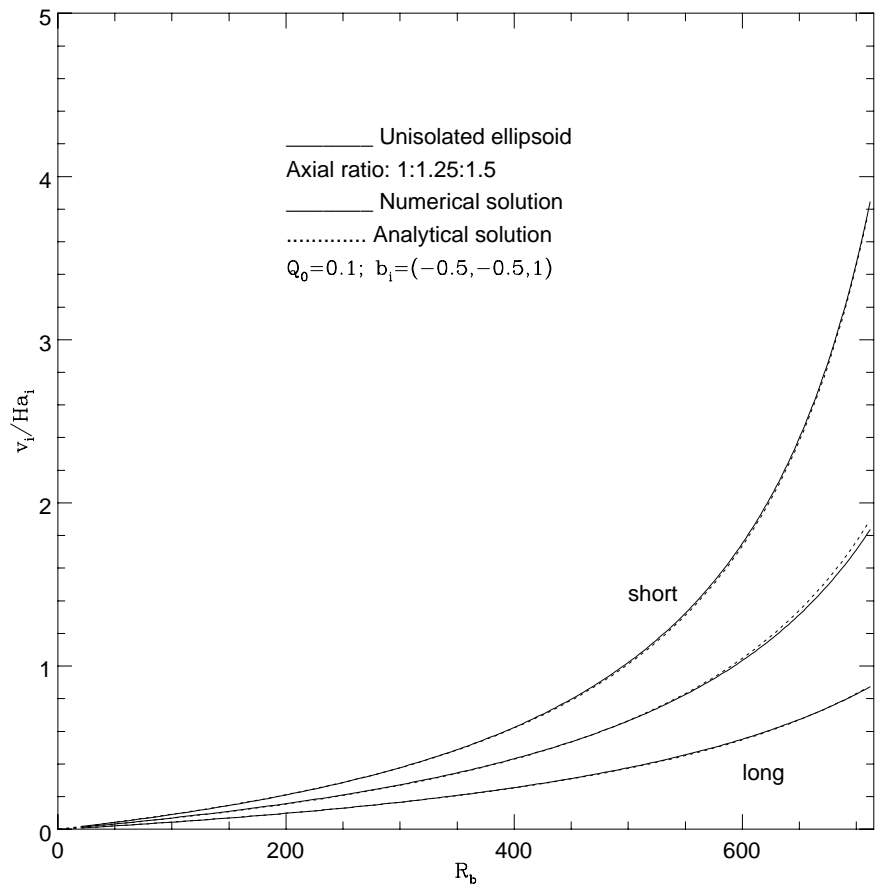

Fig. 3. a) Evolution of the axial velocity. The solid lines represent, from up to bottom, the numerical result for the peculiar velocity along the shortest, medium and longest axis. The dotted line represents the velocity obtained from Eq. (31) using the approximation for the semiaxes (Eqs. (22)-(24)). The value of the axial ratio is $1: 1.25: 1.5$, while $Q_{0}=0.1$, and $b_{i}=(-0.5,-0.5,1)$.

velocity, tends to be slowed down with increasing strength of the external field (dotted and dashed lines). The effect 


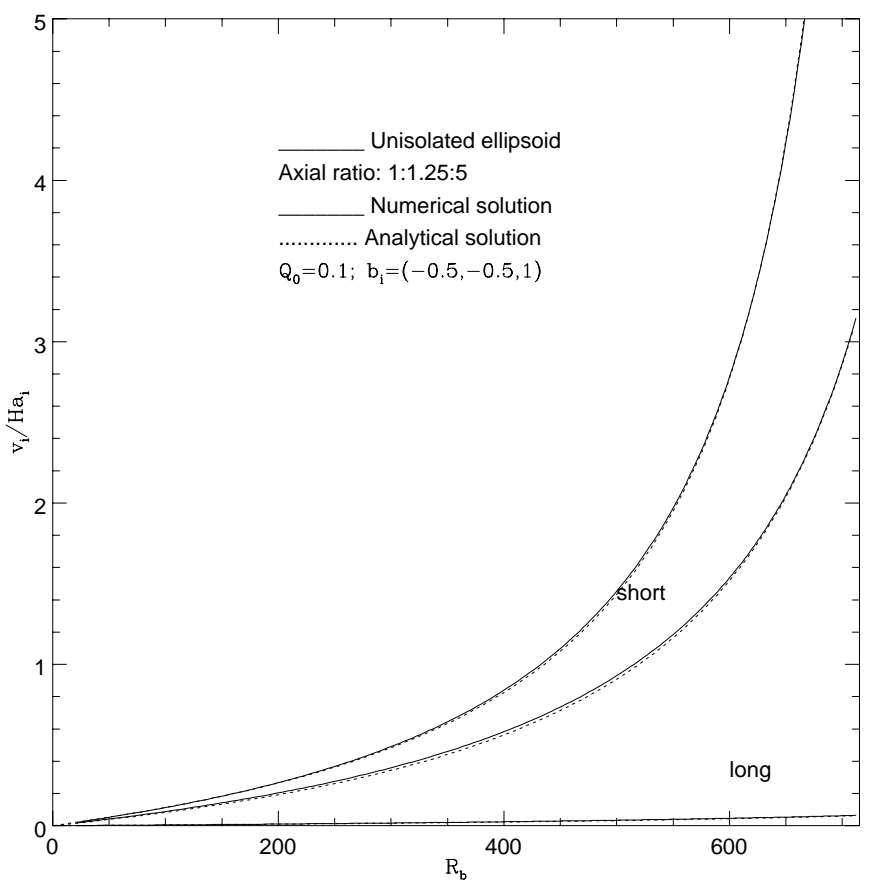

(b)

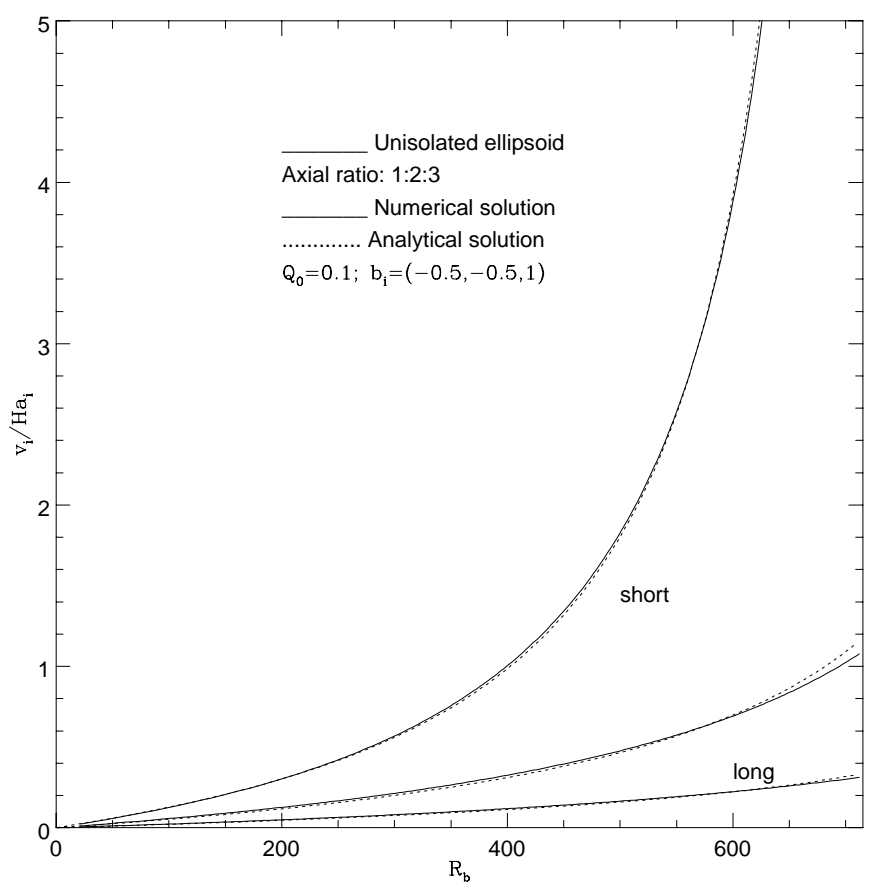

(c)

Fig. 3. b)-c) Same as Fig. 3a but now the value of the axial ratio is $1: 1.25: 5 \mathbf{b}), 1: 2: 3 \mathbf{c})$, while $Q_{0}=0.1$, and $b_{i}=(-0.5,-0.5,1)$.

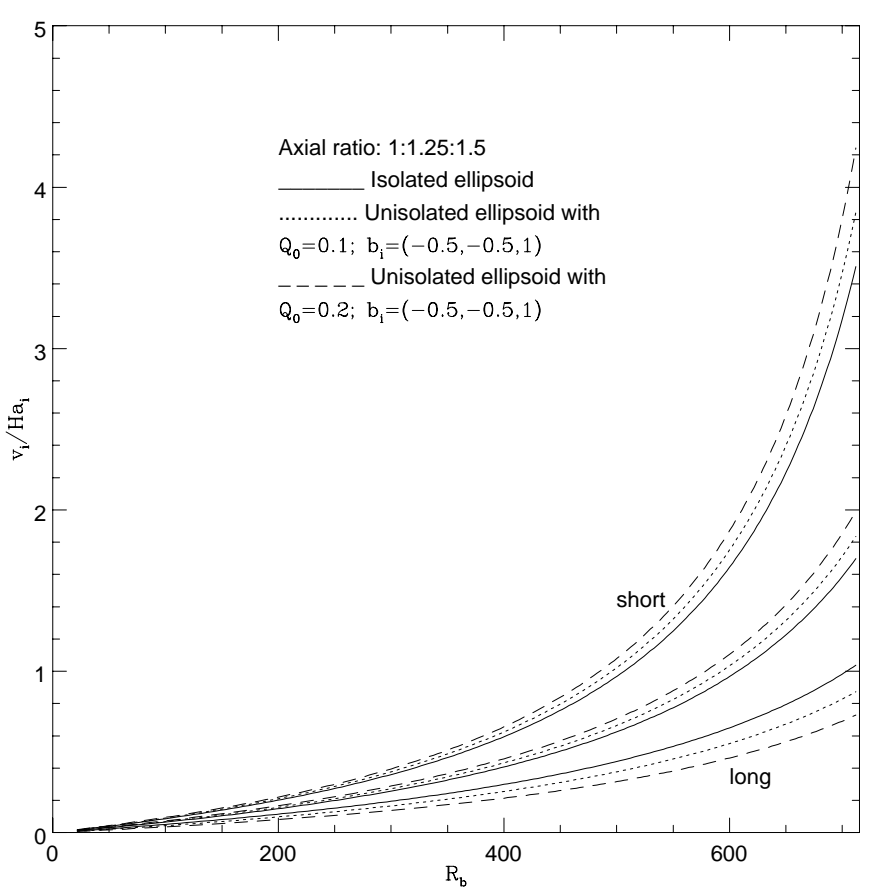

(d)

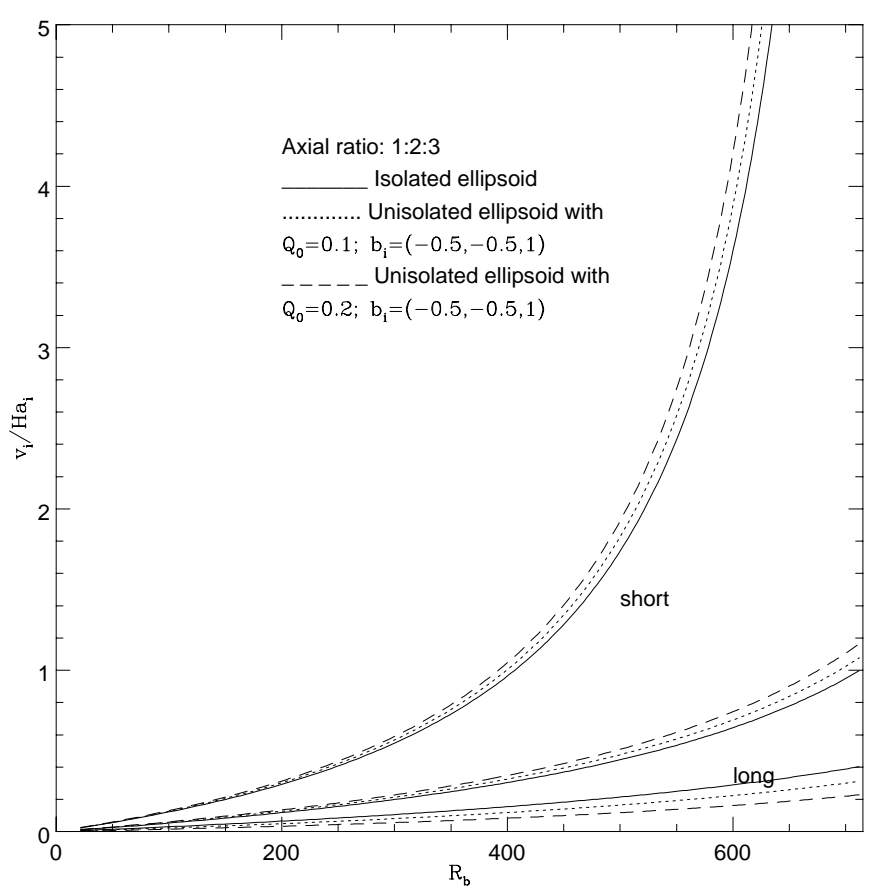

(e)

Fig. 3. d)-e) Evolution of the axial velocity. The solid line represents the peculiar velocity for an ellipsoid of axial ratio 1:1.25:1.5 d) and 1:2:3 e) when no external field is present, while the dotted and dashed lines represent the case $Q_{0}=0.1$, $b_{i}=(-0.5,-0.5,1)$, and $Q_{0}=0.2, b_{i}=(-0.5,-0.5,1)$, respectively.

of external shear on the shortest and medium axes is opposite to that on the longest one, namely shear produces an acceleration in their evolution. Figure $3 e$ is the same as the previous one, but now the axial ratio is $1: 2: 3$. Similar to the previous plot, increasing the strength of the external field produces an acceleration of evolution in the shortest and medium axes and the opposite effect on the longest one. Another feature shown by a comparison of Figs. 3d 
Table 1. Lengths of the uncollapsed axes at collapse for different values of external and internal shear.

\begin{tabular}{lll}
\hline \hline$Q_{0}$ & $a_{1}: a_{2}: a_{3}$ & $\frac{a_{3\left(t_{c}\right)}}{a_{2\left(t_{c}\right)}}$ \\
\hline 0 & $1: 1.25: 1.5$ & 1.9 \\
0.1 & $1: 1.25: 1.5$ & 2.5 \\
0.2 & $1: 1.25: 1.5$ & 3.3 \\
0.4 & $1: 1.25: 1.5$ & 5.9 \\
\hline$Q_{0}$ & $a_{1}: a_{2}: a_{3}$ & $\frac{a_{3\left(t_{c}\right)}}{a_{2\left(t_{c}\right)}}$ \\
\hline 0 & $1: 1.25: 1.7$ & 2.8 \\
0.1 & $1: 1.25: 1.7$ & 3.7 \\
0 & $1: 1.25: 2$ & 4.6 \\
0.1 & $1: 1.25: 2$ & 6 \\
\hline
\end{tabular}

and $3 \mathrm{e}$ is that the evolution of the shortest axis is accelerated with increasing asymmetry of the structure while the opposite is true for the longest axis: as noted before, external and internal shear have a qualitatively similar effect on the evolution of the shortest and longest axes. The difference between the velocities along the longest and shortest axes is larger for unisolated ellipsoids than for the isolated ones and this difference increases with increasing strength of the external field.

The evolution of the density contrast can be calculated using the usual definition:

$\delta=\frac{\rho_{\mathrm{e}}-\rho_{\mathrm{b}}}{\rho_{\mathrm{b}}}=\frac{\rho_{\mathrm{e} 0}}{\rho_{\mathrm{b} 0}} \frac{a_{10}}{a_{1}} \frac{a_{20}}{a_{2}} \frac{a_{30}}{a_{3}}\left(\frac{R_{\mathrm{b}}}{R_{\mathrm{b} 0}}\right)^{3}-1$.

As for the peculiar velocity, I calculated the evolution of the density contrast of the ellipsoid both numerically and introducing the approximate analytical solution (Eqs. (20)-(22)) in Eq. (32). The result is plotted in Figs. $4 \mathrm{a}-\mathrm{b}$. In both figures the solid lines represent the numerical results and the dotted lines the approximation, which is in good agreement with the numerical results. In Fig. $4 \mathrm{a}$, the axial ratio of the ellipsoid is $1: 1.25: 1.5$, the lines from bottom to top represent the case of an isolated ellipsoid $\left(b_{i}=(0,0,0)\right), Q_{0}=0.1, b_{i}=(-0.5,-0.5,1)$, and $Q_{0}=0.2, b_{i}=(-0.5,-0.5,1)$, respectively. The plot shows that by increasing the strength of the external field, the value of the density contrast increases. Figure $4 \mathrm{~b}$ is the same as the previous one but the configuration is less spherical, with an axial ratio 1:2:3. This last figure shows that more asymmetrical initial configurations tend to have, at a given time, larger values of $\delta$, which means that the collapse time decreases with increasing initial asymmetry. Then, internal and external shear produce a more rapid evolution of the density contrast.

\section{Overdensity at turnaround and velocity at collapse}

As reviewed in the introduction, in the literature there is not full agreement on the effect of shear on the collapse of density perturbations: while according to Bertschinger \& Jain's (1994) collapse theorem the spherical perturbations are the slowest in collapsing, several other studies conclude opposite (Peebles \& Groth 1976; Davis \& Peebles 1977; BS; Szalay \& Silk 1983; Villumsen \& Davis 1986; Peebles 1990; Bond \& Myers 1993a,b; Lokas et al. 1996; Audit et al. 1997).

In other words, the results concerning the effect of shear on collapse, are of two opposite kind:

1) Shear slows down the collapse (Peebles \& Groth 1976; Davis \& Peebles 1977; BS; Szalay \& Silk 1983; Villumsen \& Davis 1986; Peebles 1990; Bond \& Myers 1993a,b; Lokas et al. 1996; Audit et al. 1997). This result is obtained if, for example, one uses homogeneous ellipsoids to model an $e x$ tended mass distribution and not vanishing mass elements and collapse is followed even after the collapse of the first axis: the collapse of one axis is "frozen" when it becomes smaller than a certain value, and the collapse of the other axes is followed until the collapse of the third axis, which defines the collapse time. This is done to simulate the virialization process. In fact, as remarked by Eisenstein \& Loeb (1995), after the short axis collapses, it makes a small contribution to the quadrupole moment of the ellipsoid (due to the fact that the quadrupoles are proportional to the difference between the squares of the lengths of the axes) and then, in order to take into account the acquisition of angular momentum it is necessary to follow the collapse after the first axis collapse.

2) Shear accelerates the collapse (e.g., Hoffmann 1986a, 1989; Evrard \& Crone 1992; Bertschinger \& Jain 1994). This result is obtained in papers dealing with the evolution of individual mass elements for which the collapse corresponds to the collapse of the first axis.

In the remainder of this section, I shall show that, even if the effects of substructure are neglected, by approximating the structure formation by means of a homogeneous ellipsoid, and even if one assumes that the collapse is stopped when the first axis collapses, the shear slows down the rate of growth of the density contrast by lowering the peculiar velocity (WS; BS; Szalay \& Silk 1983).

To this aim, in the following, I re-derive the fundamental equations in BS for an homogeneous ellipsoid model also taking into account the external field (in BS, only the collapse of an isolated ellipsoid model was studied). These equations will be used to study the velocity at collapse and the overdensity at turnaround.

The evolution of the ellipsoid can be obtained using Eqs. (20)-(22):

$$
\begin{aligned}
& x(t)=R_{\mathrm{b}}-\frac{3}{2} \tilde{\alpha}_{1}\left(R_{\mathrm{b}}-R_{\mathrm{e}}\right)-d \times R_{\mathrm{b}}^{\left(\frac{2+3 c_{1}}{2}\right)}\left(1-\frac{3 \tilde{\alpha}_{1}}{2}\right) \\
& y(t)=R_{\mathrm{b}}-\frac{3}{2} \tilde{\alpha}_{2}\left(R_{\mathrm{b}}-R_{\mathrm{e}}\right) \\
& z(t)=R_{\mathrm{b}}-\frac{3}{2} \tilde{\alpha}_{3}\left(R_{\mathrm{b}}-R_{\mathrm{e}}\right)
\end{aligned}
$$

Using BS notation, I indicate with $x(t)=x_{0} X(t), y(t)=$ $y_{0} Y(t)$ and $z(t)=z_{0} Z(t)$, the principal axes $\left(x_{0}, y_{0}\right.$ and $z_{0}$ are the initial values of the axes). The overdensity of the 


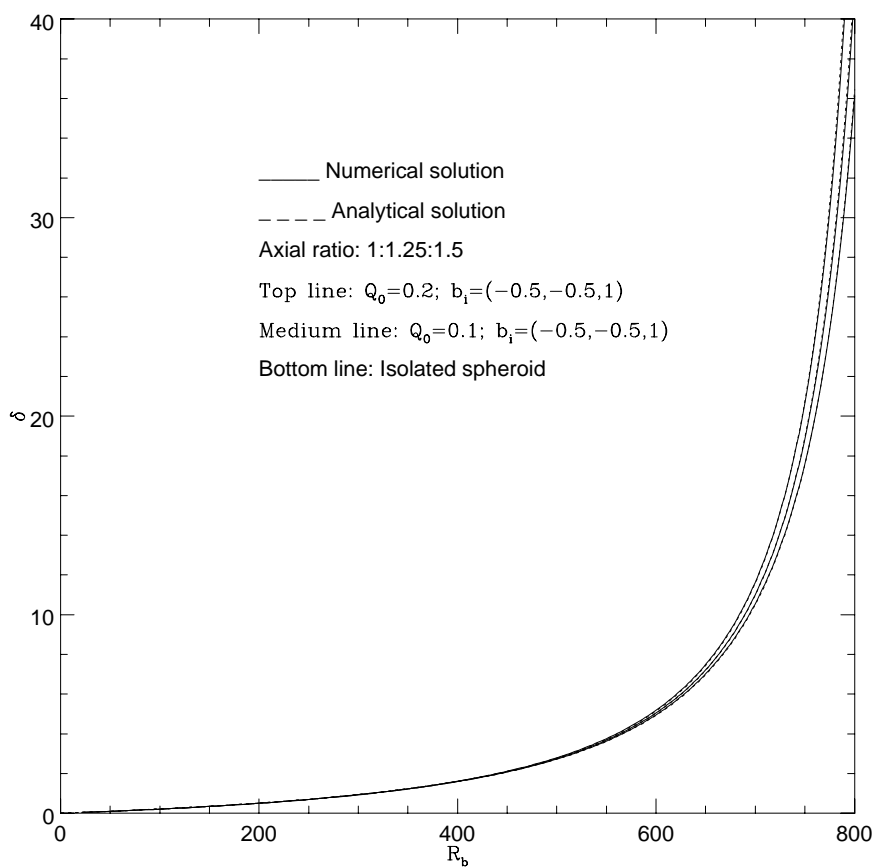

(a)

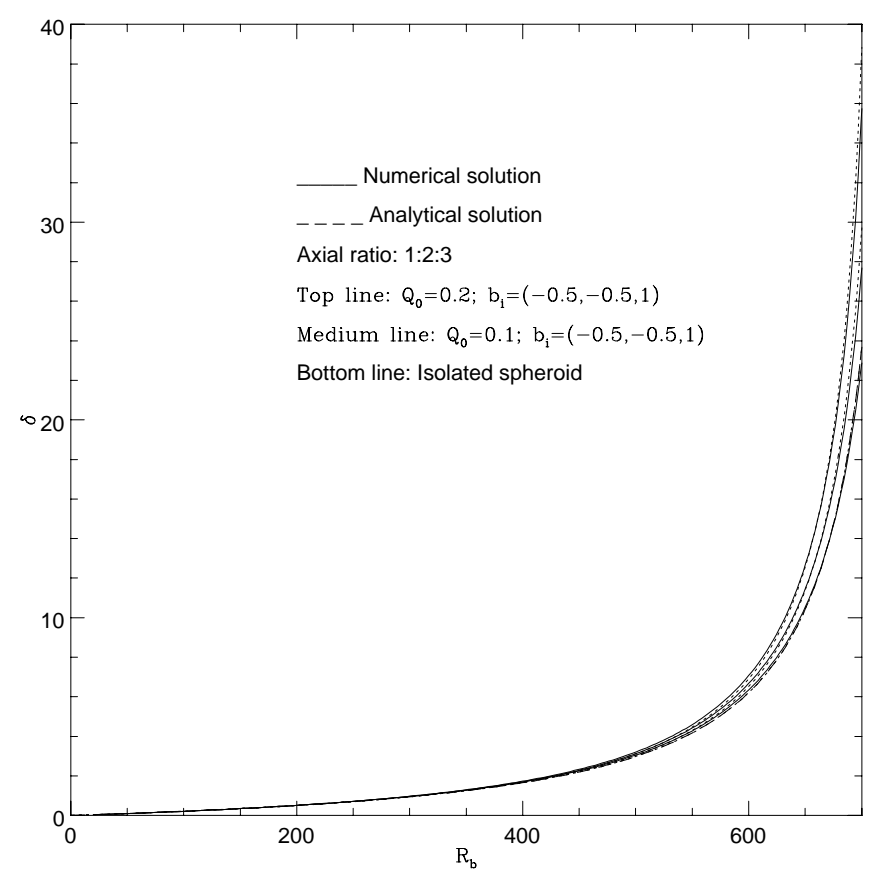

(b)

Fig. 4. a)-b) The evolution of the density contrast. The axial ratio of the ellipsoid is $1: 1.25: 1.5$ a) and $1: 2: 3$ b), the lines from bottom to top represent the case of an isolated ellipsoid $\left(b_{i}=(0,0,0)\right), Q_{0}=0.1, b_{i}=(-0.5,-0.5,1)$, and $Q_{0}=0.2$, $b_{i}=(-0.5,-0.5,1)$, respectively.

ellipsoid is the same used previously, the initial conditions are $X=Y=Z=R_{\mathrm{b}}=R_{\mathrm{e}}=1$ at $t=t_{0}$ and as before the initial velocity is equal to the Hubble velocity at $t_{0}$ (representing the initial time). The parametric equations satisfied by $R_{\mathrm{e}}(t)$ are:

$R_{\mathrm{e}}=\frac{1}{2 \delta}(1-\cos (\vartheta)), \quad \frac{t}{t_{0}}=\frac{3}{4 \delta^{3 / 2}}(\vartheta-\sin (\vartheta))$

while, since our background is an EdS universe, $R_{\mathrm{b}}(t) \propto$ $t^{2 / 3}$.

Following BS, it is easy to find that the density contrast is given by:

$$
\begin{aligned}
\delta_{i}= & \frac{R_{\mathrm{b}}^{3}}{X Y Z}-1=f_{1}^{2}(\vartheta)\left[\left(1-\frac{3 \tilde{\alpha}_{1}}{2}\right) f_{1}^{\frac{2}{3}}(\vartheta)+\frac{3}{4} \tilde{\alpha}_{1} f_{2}(\vartheta)\right. \\
& \left.-d\left(1-\frac{3 \tilde{\alpha}_{1}}{2}\right) \frac{f_{1}^{\frac{2 f}{3}}(\vartheta)}{\delta^{f-1}}\right]^{-1} \\
& \times\left[\left(1-\frac{3 \tilde{\alpha}_{2}}{2}\right) f_{1}^{\frac{2}{3}}(\vartheta)+\frac{3}{4} \tilde{\alpha}_{2} f_{2}(\vartheta)\right]^{-1} \\
& \times\left[\left(1-\frac{3 \tilde{\alpha}_{3}}{2}\right) f_{1}^{\frac{2}{3}}(\vartheta)+\frac{3}{4} \tilde{\alpha}_{3} f_{2}(\vartheta)\right]^{-1}-1
\end{aligned}
$$

where $f=\frac{2+3 c_{1}}{2}, f_{1}(\vartheta)=\frac{3}{4}(\vartheta-\sin (\vartheta))$ and $f_{2}(\vartheta)=$ $1-\cos (\vartheta)$. The density contrast at turn-around is obtained by calculating $\delta_{i}\left(\vartheta_{\mathrm{ta}}\right)$, where the parameter $\vartheta$ at the turnaround epoch is given solving the equation:

$$
\frac{2}{3 \tilde{\alpha}_{1}}=\frac{d f R_{\mathrm{b}}^{f-1}+\frac{\sin \left(\vartheta_{\mathrm{ta}}\right)}{f_{2}\left(\vartheta_{\mathrm{ta}}\right)} f_{1}^{\frac{1}{3}}-1}{d f R_{\mathrm{b}}^{f-1}-1} .
$$

Equations (37), and (38) reduce to BS Eqs. (76) and (72) for $d=0, \tilde{\alpha}_{1}=\alpha_{1}, \tilde{\alpha}_{2}=\alpha_{2}, \tilde{\alpha}_{3}=\alpha_{3}{ }^{3}$. Equation (37) yields the familiar value $\delta=(3 \pi / 4)^{2}$ in the spherical case, $\left(d=0, \tilde{\alpha}_{1}=\alpha_{1}=\tilde{\alpha}_{2}=\alpha_{2}=\tilde{\alpha}_{3}=\alpha_{3}=2 / 3\right)$. In general, in order to obtain the density contrast at turnaround, one has first to solve Eq. (38) for $\vartheta$ for an arbitrary axial ratio and substitute the value in Eq. (37). The time of turnaround can be calculated by:

$t=\frac{3 t_{0}}{4 \delta^{3 / 2}}(\vartheta-\sin (\vartheta))=\frac{t_{\mathrm{ff}}}{2 \pi}(\vartheta-\sin (\vartheta))$

where $t_{\mathrm{ff}}$ is the free-fall time:

$t_{\mathrm{ff}}=\frac{3 \pi}{2 \delta^{3 / 2}} t_{0}$.

In Fig. 5, I plotted the density contrast at turn-around for a prolate spheroid for several values of the longest axis, $a_{3}$, (the other two axes have fixed value $a_{1}: a_{2}=1: 1$ ) (note that from now on, and in the figures, $a_{\mathrm{i}}$ represents the initial value of the $i$ th axis). The solid lines, from top to bottom, represent numerical results for the density contrast for an isolated spheroid $\left(b_{i}=(0,0,0)\right)$, and for unisolated spheroids with $Q_{0}=0.1, b_{i}=(-0.5,-0.5,1)$ and $Q_{0}=0.2,(-0.5,-0.5,1)$, respectively. The dashed lines represent the approximate solution (Eq. (37)). The figure shows that the density contrast at turn-around is reduced, relative to the value $\delta=(3 \pi / 4)^{2}$ typical of spherical collapse when $a_{3}>1$. This reduction increases with

\footnotetext{
3 Some equations in BS contains some typographical misprints, for example Eqs. (76) and (80).
} 


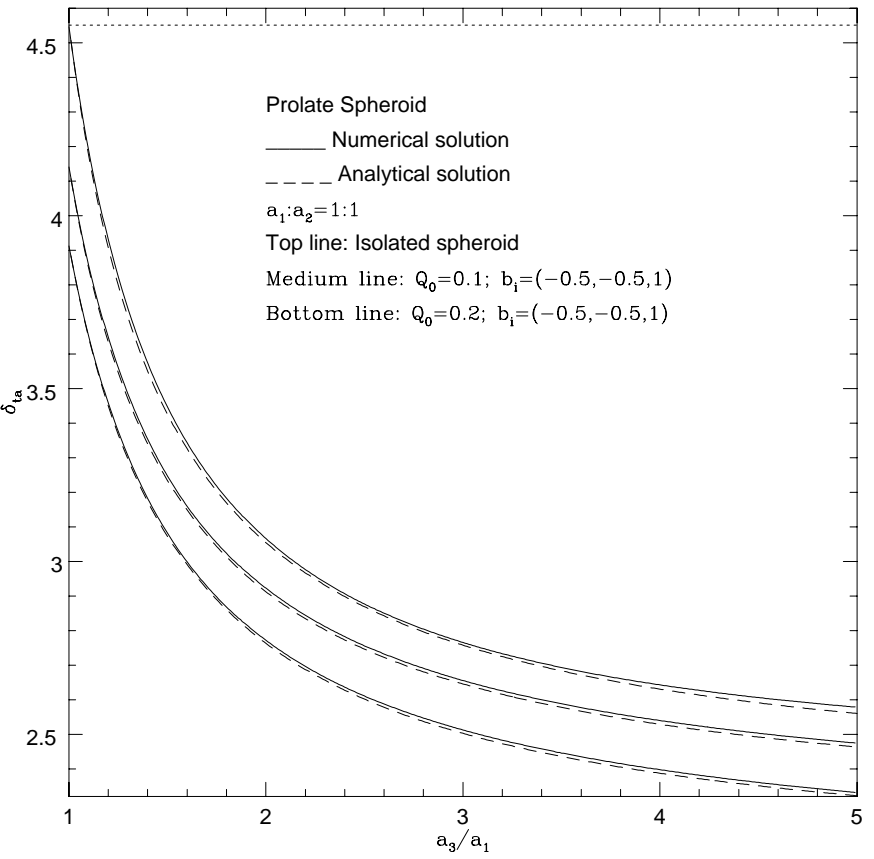

Fig. 5. The density contrast at turnaround for a prolate spheroid for several values of the longest axis, $a_{3}$, (the other two axes have fixed value $a_{1}: a_{2}=1: 1$ ). The solid lines, from top to bottom, represent numerical results for the density contrast for an isolated spheroid $\left(b_{i}=(0,0,0)\right)$, and for unisolated spheroids with $Q_{0}=0.1, b_{i}=(-0.5,-0.5,1)$ and $Q_{0}=0.2$, $(-0.5,-0.5,1)$, respectively. The dashed lines represent the approximate solution (Eq. (37)). The upper dotted line represents the value of the density contrast at turnaround for a spherical perturbation.

increasing initial asymmetry of the spheroid. Although not shown, the reduction is larger for oblate spheroids: in this case, for extreme initial flattening (8:8:1) the value of $\delta_{\text {ta }}$ can be reduced to values as small as $\simeq 1$. The effect of the external field is to reduce the value of $\delta_{\text {ta }}$ even more. An interesting question that can arise because of the previous result is the following: if at turn-around the density contrast predicted by the ellipsoid model can be a factor of 3 smaller than that of the spherical model, then one should expect that the difference between the two models starts at $\delta<(3 \pi / 4)^{2}$. Does the prediction of these two models match the linear theory? In order to answer this question, I compared the value of $\delta$ given by the ellipsoid model to that of the spherical one. The result is plotted in Fig. 6. The solid line represents the density contrast predicted by the spherical model, while the dotted, short-dashed, long-dashed and long-dashedshort-dashed lines represents the prediction of the ellipsoid model with $Q_{0}=0.1, b_{i}=(-0.5,-0.5,1)$ and initial axial ratio $1: 1.25: 1.5,1: 1.25: 3,1: 1.25: 5,1: 1.25: 8$, respectively. As shown, the difference between the prediction of spherical and ellipsoid model starts at $\delta>1$. In Fig. 7, the same calculation is repeated for an oblate spheroid. Here, the solid line represents the density contrast predicted by the spherical model, while the dotted, short-dashed, longdashed lines represents the prediction of the model with

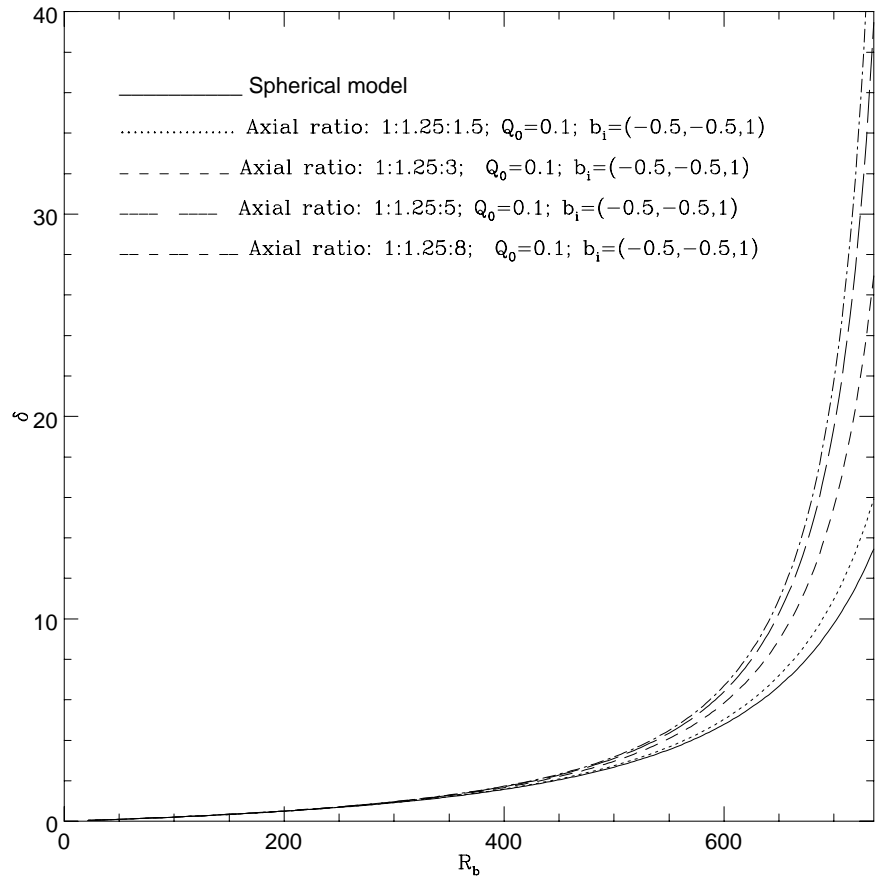

Fig. 6. Comparison between density contrast predicted by spherical and ellipsoidal model. The solid line represents the density contrast predicted by the spherical model, while the dotted, short-dashed, long-dashed and long-dashed-shortdashed lines represents the prediction of the ellipsoid model with $Q_{0}=0.1, b_{i}=(-0.5,-0.5,1)$ and initial axial ratio $1: 1.25: 1.5,1: 1.25: 3,1: 1.25: 5$, and $1: 1.25: 8$ respectively.

$Q_{0}=0.1, b_{i}=(-0.5,-0.5,1)$ and initial axial ratio $2: 2: 1$, $4: 4: 1,8: 8: 1$, respectively. In this case the two models start to give different predictions at $\delta \simeq 0.6$. So in both cases, the two models are different only when we are far away from the linear regime.

The density contrast at virialization is a bit more difficult to calculate. To begin with, it is important to recall the difference between virialization and collapse: this last term describe a state of the system in which the density approaches infinity, while virialization is characterized by $|U|=2 K$, where $U$ and $K$ are, respectively, the potential and kinetic energy. Only in the case of perfectly spherical infall, are collapse and virialization synonymous (although in the case of a bound system one rapidly follows the other). In this case, the infall cannot be halted and it proceeds towards a singularity, with all mass of the system collapsing to a single point which means that the density contrast becomes infinite. This result is physically unacceptable and to prevent the system from reaching this state it is necessary to introduce, by hand, the assumption that the collapse halts when spherical shells reach a particular radius $r_{\mathrm{f}}=r_{\mathrm{v}}=r_{\mathrm{ta}} / 2$, (where $r_{\mathrm{f}}, r_{\mathrm{v}}$ and $r_{\mathrm{ta}}$ are the final radius, the virialization radius and the turnaround radius, respectively $)^{4}$. Then, in the spherical infall model, the density contrast of the virializing structure is

\footnotetext{
${ }^{4}$ This major drawback of the spherical model is removed by taking account of the angular momentum in the equation of motion (see Engineer et al. 2000).
} 


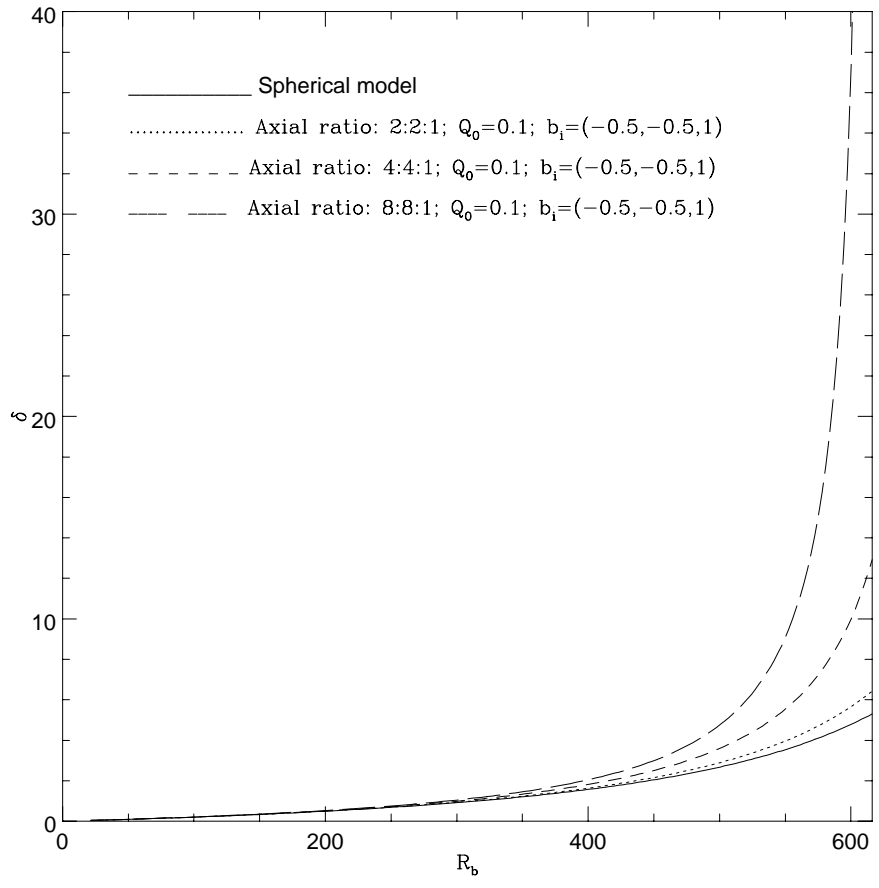

Fig. 7. Comparison between density contrast predicted by spherical and oblate spheroidal models. The solid line represents the density contrast predicted by the spherical model, while the dotted, short-dashed, and long-dashed lines represents the prediction of the model with $Q_{0}=0.1, b_{i}=$ $(-0.5,-0.5,1)$ and initial axial ratio $2: 2: 1,4: 4: 1,8: 8: 1$, respectively.

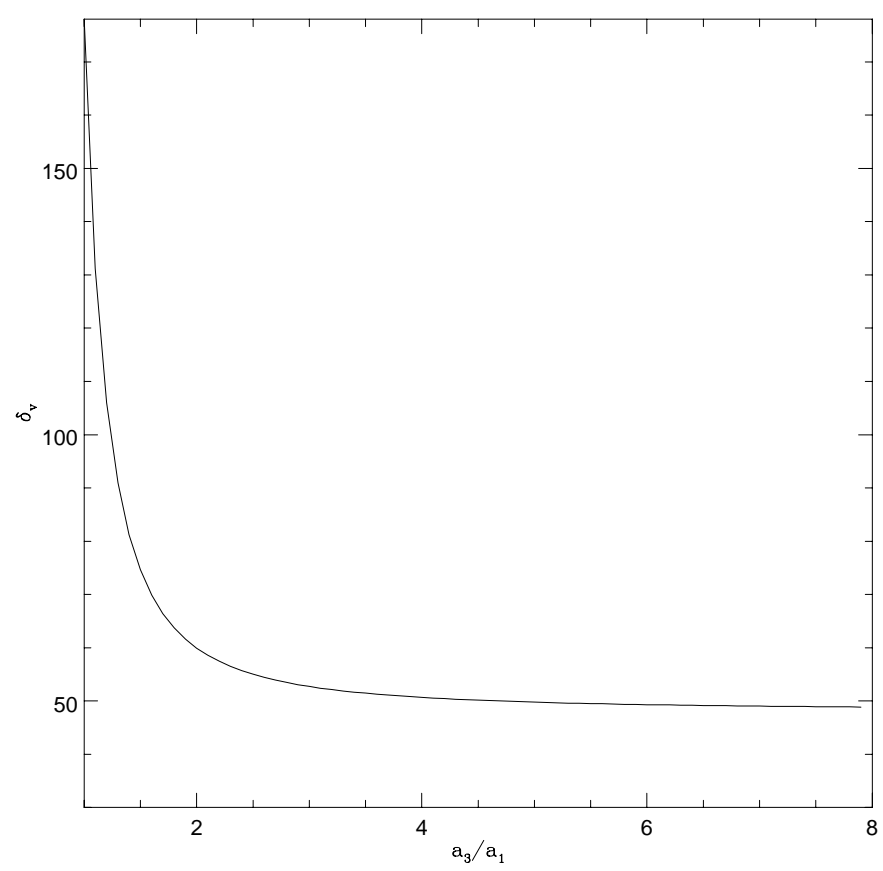

Fig. 8. Density contrast at virialization. The solid line refers to an isolated prolate spheroid.

calculated assuming that, after the shell collapses, the final radius is $r_{\mathrm{f}}=r_{\mathrm{v}}=r_{\mathrm{ta}} / 2$ : this leads to the conclusion that $\delta_{\mathrm{v}}=178$. The density contrast of a non-spherical

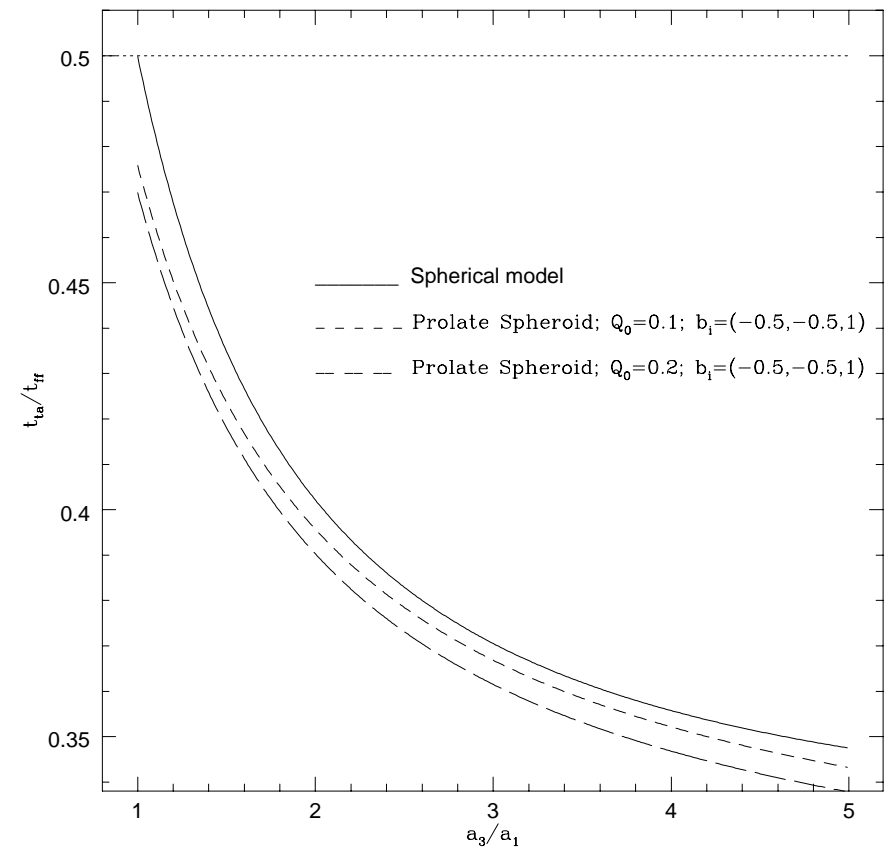

Fig. 9. Turnaround epoch for a prolate spheroid. The solid, short-dashed and long-dashed lines, represent respectively the time of turnaround for an isolated spheroid and for spheroids having $Q_{0}=0.1, b_{i}=(-0.5,-0.5,1)$ and $Q_{0}=0.2$, $(-0.5,-0.5,1)$. The upper dotted line represents the value of the density contrast at turnaround for a spherical perturbation.

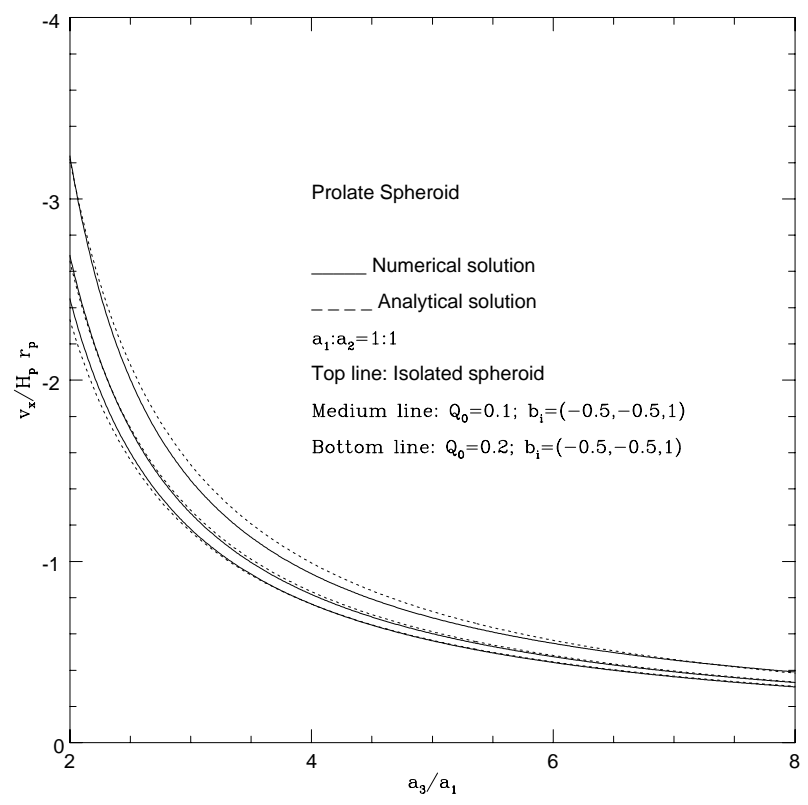

Fig. 10. Axial velocity at collapse as function of the ratio of the initial value of the axes, $a_{3} / a_{1}$. The solid lines represent the numerical results of the collapse velocity for a prolate spheroid, the dotted lines the result obtained from the approximate solution. The top curve represents the velocity for an isolated spheroid, the medium one the same quantity for a spheroid having $Q_{0}=0.1, b_{i}=(-0.5,-0.5,1)$, and the last bottom curve the velocity for a spheroid having $Q_{0}=0.2$, and $b_{i}=(-0.5,-0.5,1)$. 
virialized system can be calculated using the same definition given for the spherical system, namely:

$\delta_{\mathrm{v}}=\frac{R_{\mathrm{b}}^{3}\left(t_{\mathrm{c}}\right)}{X\left(t_{1 / 2}\right) Y\left(t_{1 / 2}\right) Z\left(t_{1 / 2}\right)}-1$

where $t_{1 / 2}$ is the time at which the shortest axis has a length $1 / 2$ of the value at maximum expansion, $x\left(t_{1 / 2}\right)=$ $x_{t a} / 2$ (see Peebles 1990).

The time at collapse of the shortest axis can be obtained by means of the second part of Eq. (36) once the parameter $\vartheta_{\mathrm{c}}$ at the collapse time, characterized by $X\left(t_{\mathrm{c}}\right)=0$, is known. After some calculations it is possible to show that $\vartheta_{\mathrm{c}}$ can be obtained by solving the following equation:

$$
\frac{2}{3 \tilde{\alpha}_{1}}=\frac{\frac{1-\cos \left(\vartheta_{\mathrm{c}}\right)}{2} f_{1}^{\frac{-2}{3}}\left(\vartheta_{\mathrm{c}}\right)+d\left(\frac{f_{1}^{\frac{2}{3}}\left(\vartheta_{\mathrm{c}}\right)}{\delta}\right)^{f-1}-1}{d\left(\frac{f_{1}^{\frac{2}{3}}\left(\vartheta_{\mathrm{c}}\right)}{\delta}\right)^{f-1}-1}
$$

which, for $d=0$ and $\tilde{\alpha}_{1}=\alpha_{1}$, reduces to Eq. (78) of BS. The time $t_{1 / 2}$, or equivalently, $\vartheta_{1 / 2}$, is obtained solving the equation:

$x\left(t_{1 / 2}\right)=x_{\mathrm{ta}} / 2$

where $x(t)$ is given in Eq. (33) and $x_{\mathrm{ta}}$ is obtained using again Eq. (33) and calculating $x\left(\vartheta_{\mathrm{ta}}\right)$. The result is plotted in Fig. 8. The solid line shows that, with increasing initial anisotropy, the density contrast decreases from the value $\delta_{\mathrm{v}}=178$, for the spherical case, to a value of $\delta_{\mathrm{v}} \simeq 48$, for large initial "flattening" (semiaxes ratio 1:1:8). The result plotted refers to an isolated prolate spheroid, while for the oblate case and for the same axial ratio $(8: 8: 1) \delta_{\mathrm{v}} \simeq 11$. These results are in qualitative agreement with Peebles (1990).

The results in Figs. 5-8 could seem in disagreement with Figs. $4 \mathrm{a}-\mathrm{b}$, since in those figures the value of $\delta$ increases with increasing initial asymmetry of the ellipsoid. The reason why $\delta_{\text {ta }}$ decreases with increasing initial asymmetry is due to the fact that the turn-around $t_{\mathrm{ta}}$, and also the collapse epoch, moves towards lower values of time, $t$, for larger values of initial asymmetry of a given perturbation and larger strength of the external field. This last effect is shown in Fig. 9: the solid, short-dashed and long-dashed lines, represent respectively the time of turn-around for an isolated spheroid and for spheroids having $Q_{0}=0.1, b_{i}=(-0.5,-0.5,1)$ and $Q_{0}=0.2$, $(-0.5,-0.5,1)$. More asymmetric structures are characterized by a smaller value of turn-around time, and the external field contributes to this reduction of $t_{\mathrm{ta}}$. In the case of oblate spheroids, the collapse time can be reduced to values $t \simeq 0.3 t_{\text {ff }}$ for initial axial ratio $8: 8: 1$.

Another interesting quantity that can be obtained is the collapse velocity at the time of collapse. I shall calculate the collapse velocity using the same steps followed by $\mathrm{BS}$, with the difference that the spheroids considered in the following are prolate. Their evolution is obtained as before, putting Eqs. (33)-(35) in the equation for the collapse velocity down the shortest axis:

$v_{x}=x_{0} \dot{X}$.

Calculating the derivative of Eq. (33) and inserting it in Eq. (44), I find that:

$$
\begin{aligned}
v_{x \mathrm{c}}= & -x_{0} \frac{3}{2} \tilde{\alpha}_{1} \dot{R}_{\mathrm{b}}\left[1-\frac{2}{3 \tilde{\alpha}_{1}}-\left(\frac{3}{4}\right)^{\frac{1}{3}}\right. \\
& \left.\times \frac{\sin \left(\vartheta_{\mathrm{c}}\right)\left(\vartheta_{\mathrm{c}}-\sin \left(\vartheta_{\mathrm{c}}\right)\right)}{1-\cos \left(\vartheta_{\mathrm{c}}\right)}+d f R_{\mathrm{b}}^{f-1}\left(\frac{2}{3 \tilde{\alpha}_{1}}-1\right)\right]
\end{aligned}
$$

here the subscript "c" means that the corresponding quantity is calculated at the collapse epoch. Equation (45) can be written in a different form using the following equations:

$$
\begin{aligned}
Z_{\mathrm{c}}= & R_{\mathrm{bc}}-\frac{3 \tilde{\alpha}_{3}}{2}\left(R_{\mathrm{bc}}-R_{\mathrm{ec}}\right)=R_{\mathrm{bc}}\left(1-\frac{\tilde{\alpha}_{3}}{\tilde{\alpha}_{1}}\right) \\
& +\frac{3 \tilde{\alpha}_{3}}{2} d\left(\frac{2}{3 \tilde{\alpha}_{1}}-1\right) R_{\mathrm{b}}^{f} \simeq R_{\mathrm{bc}}\left(1-\frac{\tilde{\alpha}_{3}}{\tilde{\alpha}_{1}}\right)
\end{aligned}
$$

(see Eq. (79) of BS). Approximating Eq. (42) by means of Eq. (78) of BS:

$1-\frac{2}{3 \tilde{\alpha}_{1}}=\frac{1-\cos \left(\vartheta_{\mathrm{c}}\right)}{2}\left[\frac{3}{4}\left(\vartheta_{\mathrm{c}}-\sin \left(\vartheta_{\mathrm{c}}\right)\right)\right]^{\frac{-2}{3}}$

and approximating the condition $x(t)=0$ (see Eq. (33)), defining the collapse time, with the Eq. (77) of BS:

$\left(1-\cos \left(\vartheta_{\mathrm{c}}\right)\right) / 2 \delta=R_{\mathrm{bc}}\left(1-\frac{2}{3 \alpha_{1}}\right)$.

Finally I get:

$$
\begin{aligned}
v_{x \mathrm{c}}= & -\left[\frac{\dot{R}_{\mathrm{bc}}}{R_{\mathrm{bc}}} r_{\mathrm{c}}\right] \frac{3 \tilde{\alpha}_{1}-2}{\tilde{\alpha}_{1}-\tilde{\alpha}_{3}}\left\{1-\frac{3}{2} \frac{\sin \left(\vartheta_{\mathrm{c}}\right)\left(\vartheta_{\mathrm{c}}-\sin \left(\vartheta_{\mathrm{c}}\right)\right)}{\left(1-\cos \left(\vartheta_{\mathrm{c}}\right)\right)^{2}}\right. \\
& +2 d f\left[\frac{1-\cos \left(\vartheta_{\mathrm{c}}\right)}{2 \delta\left(1-\frac{2}{3 \tilde{\alpha}_{1}}\right)}\right]^{f-1}\left(\frac{2}{3 \tilde{\alpha}_{1}}-1\right) \\
& \left.\times \frac{\left[\frac{3}{4}\left(\vartheta_{\mathrm{c}}-\sin \left(\vartheta_{\mathrm{c}}\right)\right)\right]^{\frac{2}{3}}}{1-\cos \left(\vartheta_{\mathrm{c}}\right)}\right\} \frac{x_{0}}{z_{0}} \tilde{\alpha}_{1}
\end{aligned}
$$

where $r_{\mathrm{c}}=z_{0} Z_{\mathrm{c}}$ is the radius of the collapsed object and $R_{\mathrm{bc}}$ represents $R_{\mathrm{b}}\left(t_{\mathrm{c}}\right)$. Using Eq. (49) instead of Eq. (45) introduces errors of order $\simeq 10 \%$. In the case of a prolate spheroid, one has that:

$$
\begin{aligned}
v_{x \mathrm{c}}= & -\left[\frac{\dot{R}_{\mathrm{bc}}}{R_{\mathrm{bc}}} r_{\mathrm{c}}\right] \frac{3 \tilde{\alpha}_{1}-2}{\tilde{\alpha}_{1}-\tilde{\alpha}_{3}}\left\{1-\frac{3}{2} \frac{\sin \left(\vartheta_{\mathrm{c}}\right)\left(\vartheta_{\mathrm{c}}-\sin \left(\vartheta_{\mathrm{c}}\right)\right)}{\left(1-\cos \left(\vartheta_{\mathrm{c}}\right)\right)^{2}}\right. \\
& +2 d f\left[\frac{1-\cos \left(\vartheta_{\mathrm{c}}\right)}{2 \delta\left(1-\frac{2}{3 \tilde{\alpha}_{1}}\right)}\right]^{f-1}\left(\frac{2}{3 \tilde{\alpha}_{1}}-1\right) \\
& \left.\times \frac{\left[\frac{3}{4}\left(\vartheta_{\mathrm{c}}-\sin \left(\vartheta_{\mathrm{c}}\right)\right)\right]^{\frac{2}{3}}}{1-\cos \left(\vartheta_{\mathrm{c}}\right)}\right\} \sqrt{1-e^{2}} \tilde{\alpha}_{1}
\end{aligned}
$$


where I have defined $1-e^{2}=\left(x_{0} / z_{0}\right)^{2}$. In the case of an oblate spheroid:

$$
\begin{aligned}
v_{z \mathrm{c}}= & -\left[\frac{\dot{R}_{\mathrm{bc}}}{R_{\mathrm{bc}}} r_{\mathrm{c}}\right] \frac{3 \tilde{\alpha}_{3}-2}{\tilde{\alpha}_{3}-\tilde{\alpha}_{1}}\left\{1-\frac{3}{2} \frac{\sin \left(\vartheta_{\mathrm{c}}\right)\left(\vartheta_{\mathrm{c}}-\sin \left(\vartheta_{\mathrm{c}}\right)\right)}{\left(1-\cos \left(\vartheta_{\mathrm{c}}\right)\right)^{2}}\right. \\
& +2 d f\left[\frac{1-\cos \left(\vartheta_{\mathrm{c}}\right)}{2 \delta\left(1-\frac{2}{3 \tilde{\alpha}_{3}}\right)}\right]^{f-1}\left(\frac{2}{3 \tilde{\alpha}_{3}}-1\right) \\
& \left.\times \frac{\left[\frac{3}{4}\left(\vartheta_{\mathrm{c}}-\sin \left(\vartheta_{\mathrm{c}}\right)\right)\right]^{\frac{2}{3}}}{1-\cos \left(\vartheta_{\mathrm{c}}\right)}\right\} \sqrt{1-e^{2}} \tilde{\alpha}_{3}
\end{aligned}
$$

having defined $1-e^{2}=\left(z_{0} / x_{0}\right)^{2}$ and $r_{\mathrm{c}}=x_{0} X_{\mathrm{c}}$. If $d=0$, $\tilde{\alpha}_{1}=\alpha_{1}=\tilde{\alpha}_{2}=\alpha_{2}=\tilde{\alpha}_{3}=\alpha_{3}=2 / 3$, the spheroid is isolated and Eqs. (82)-(83) of BS is recovered.

In Fig. 10, I plot $\frac{v_{x c}}{H_{c} r_{c}}$, as a function of the ratio of the initial value of the axes, $a_{3} / a_{1}$. The solid lines represent numerical results of the collapse velocity for a prolate spheroid $\left(a_{1}=a_{2}<a_{3}\right)$, the dotted lines represent the result obtained from the approximate solution. The top curve represents the velocity for an isolated spheroid, the medium one the same quantity for a spheroid having $Q_{0}=0.1, b_{i}=(-0.5,-0.5,1)$, and the bottom curve the velocity for a spheroid having $Q_{0}=0.2$, $b_{i}=(-0.5,-0.5,1)$.

The figure shows two trends:

a) the collapse velocity is reduced with increasing initial asymmetry. For example for $a_{1} / a_{3}=0.3$ the collapse velocity is reduced to the Hubble velocity in the plane of collapse (plane of the pancake for oblate spheroids ) $\left(H_{\mathrm{c}} r_{\mathrm{c}}\right)$, while in the case of more extreme "flattening" $a_{1} / a_{3}=0.125$, the collapse velocity is reduced by a factor of $\simeq 2.5$ with respect to the previous value. In the case of oblate spheroids, for this initial asymmetry, this value is $\simeq 6$.

b) The collapse velocity is reduced with increasing strength of the external field. In the case of the bottom curve, for $a_{1} / a_{3}=0.125$, the collapse velocity is reduced by a factor of $\simeq 3$. Similar to item (a), in the case of oblate spheroids, for this last initial asymmetry, this value can be larger than $\simeq 6$.

In other words, the slowing down of the rate of growth of density contrast produces a lowering of the peculiar velocity in qualitative and quantitative agreement with BS and Szalay \& Silk (1983).

The results obtained help to clarify the controversy relative to the previrialization conjecture. According to this paper and with WS and BS and in agreement with Hoffman (1986a) and Bertschinger \& Jain's collapse theorem, it is surely true that the effect of the shear is to reduce the collapse time of perturbations. As remarked in item "b" of Sect. 3 and in agreement with WS and BS: The collapse time of perturbations of fixed initial overdensity, for a fixed background, decreases with increasing initial asymmetry.

The decrease of the collapse time compensates the effect of the increase of density contrast and collapse velocity produced by the shear. To be more clear, it is useful to concentrate in Figs. 3, 4. If the collapse of the ellipsoids occurred at a fixed value of $t$, just like for the spherical model $\left(t=0.5 t_{\mathrm{ff}}\right)$, the larger increase in density contrast or velocity produced by the initial asymmetry, in comparison with the spherical model, should have as a result that at collapse both the density contrast and velocity should be enhanced (with respect to the spherical model). But in the ellipsoidal collapse, the collapse time decreases with increasing initial anisotropy, and as we have previously seen, in case of initial axial ratio 8:8:1 it is given by $t \simeq 0.3 t_{\mathrm{ff}}$. As a consequence, the values of density contrast and collapse velocity at collapse time are always reduced with respect to the spherical collapse, in agreement with WS, BS, Szalay \& Silk (1983).

I also add that in the real collapse other effects have an important role, (e.g., the effects of small scale substructure). Both the Hoffman (1986a) and Bertschinger \& Jain (1994) results are valid for a fluid element, that has no substructure by definition, while a small scale substructure produces a slowing down of the collapse at least in two ways:

1) encounters between infalling clumps and substructure internal to the perturbation (Antonuccio-Delogu \& Colafrancesco 1994; Del Popolo \& Gambera 1997; Del Popolo \& Gambera 1999);

2) tidal interaction of the main proto-structure with substructure external to the perturbation (Peebles 1990; Del Popolo \& Gambera 1998).

Moreover, it should be pointed out that, as more smallscale power is present, the collapse of a perturbation may be slowed down in a way that could inhibit the effect of shear.

Similar to Bertschinger \& Jain (1994), the model presented in this paper does not take account of the substructure internal to the system. I, however, recall that the same shortcoming was present in Peebles (1990): in that paper the substructure was suppressed, since it adopted a homogeneous Poisson distribution of particles within the protocluster (Peebles 1990). This limit has the effect of underestimating the effect of previrialization, (Peebles 1990). In other words, the slowing down of the collapse obtained in this paper (similarly to that of Peebles 1990) is smaller than that we would find if we had used a system having internal substructure, as in the above point 1 .

Before concluding, I want to discuss the impact of the results of this paper on our view of structure formation.

The reduction of the rate of growth of overdensity and collapse velocity has several consequences for structure formation. A first consequence is a change of the mass function, the two-point correlation function, and the mass that accretes on density peaks. These last consequences are connected to the effects of the shear (Audit et al. 1997, 1998). According to Audit et al. (1997, 1998), the mass function depends on two parameters, a density threshold $\delta_{\mathrm{c}}$ and a shear threshold $\sigma_{\mathrm{c}}$. According to the previous authors, structures results from a collapse along their third principal axis, which is slowed down by the effect of the shear (in agreement with our results). Therefore on small 
scales, where the shear is statistically greater, structures need on average a higher density contrast to collapse and as a consequence the number of objects with $\sigma(M) \leq 1^{5}$ decreases as compared to the collapse of the second or first axis, and so the mass function is much below the standard Press-Shechter prediction. Even the two-point correlation function of galaxies and clusters of galaxies are strongly modified since the two-point correlation function of the collapsed halos is directly connected to the number of objects of a given mass (see Peebles 1993; Sheth \& Jain 1997; Del Popolo \& Gambera 1999; Del Popolo et al. 1999). Another important consequence of the results described is connected to the value of the density parameter, $\Omega$. Since the initial anisotropy (internal shear) and the tidal interaction with external objects (external shear), slows down the collapse infalling velocity, when using the spherical infall model we underestimate the value of the density parameter (Szalay \& Silk 1983; Lee et al. 1986; Taruya \& Soda 2000). The previously described effects are even larger if the structure acquires angular momentum during evolution (Del Popolo \& Gambera 1999; Del Popolo \& Gambera 2000). In our model, we assumed that the principal axes of the external tidal tensor are always oriented along the principal axes of the mass tensor and this implies that the linear angular momentum should be zero (at least from the linear phase to shell-crossing), and so while it is legitimate to speak about the effects of shear on structure formation, we do not take account of the effects of angular momentum acquisition on structure formation, at least before gravitational collapse ${ }^{6}$. As a result, since tidal forces produces effects similar to that due to shear, the quoted limit has the consequence of underestimating the global effect on structure formation of the interaction of a protostructure with neighboring ones (see also Del Popolo \& Gambera 1999; Del Popolo \& Gambera 2000).

Finally, we want to recall, as previously reported, that in the case of an initial spherical configuration our model reduces to Watanabe's. In this case, the misalignment condition is verified and the sphere can acquire angular momentum.

\section{Conclusions}

I examined the effect of internal and external shear on the evolution of non-spherical inhomogeneities in a EdS universe. The study was based upon an approximate analytical solution of the equation of motions of the axes of the

\footnotetext{
${ }^{5} \sigma(M)$ is the mass variance.

${ }^{6}$ However, since the collapse of a protostructure is a violent phenomenon, the conditions of Kelvin's circulation theorem should be violated (Chernin 1970). This leads to the acquisition of vorticity by the formation of shock fronts in the protostructure (pancake), at the time of shell-crossing (Doroshkevich 1970). Analytical studies by Pichon \& Bernardeau (1999) have also shown that vorticity generation becomes significant at the scales of 3-4 $h^{-1} \mathrm{Mpc}$, and increases with decreasing scale.
}

ellipsoid. In the first part of the paper, I found the analytical solution to the quoted equations and I compared the result with the numerical solution of Icke's (1973) equations, and in the case of isolated ellipsoids with the WS analytical solution. The analytical approximation is in good agreement with the numerical results both for isolated and unisolated ellipsoids, and it gives a better approximation to the numerical results with respect to the WS analytical solution. The quoted solution was used to study the effect of shear on the density contrast and peculiar velocity. The results show that:

a) The collapse time of perturbations of fixed initial overdensity, for a fixed background, decreases with increasing initial asymmetry and strength of the external field. To be more precise, the evolution of the shortest axis is accelerated with increasing asymmetry of the structure while the opposite is true for the longest axis. The effect of a positive tidal force, along a given axis, is that of slowing down the expansion of the ellipsoid along this axis and the effect of a negative tidal field is that of accelerating the expansion of the ellipsoid along that axis: external and internal shear have a qualitatively similar effect on the evolution of the shortest and longest axes.

b) The difference between the velocities along the longest and shortest axes is larger for unisolated ellipsoids than for the isolated ones and this difference increases with increasing strength of the external field.

c) While for isolated ellipsoids or for unisolated ones with small values of $|\gamma|$, the axial ratio does not appreciably change until the perturbation enters a strongly nonlinear regime, (the self-gravity is dominant); for large values of $|\gamma|$, the collapse is anisotropic even for not large values of $\delta$. This means that the collapsing region geometry is strongly influenced by the external shear: if the external field is strong enough, then external shear is dominant (with respect to internal shear) in shaping the region, in agreement with Eisenstein \& Loeb (1995) and Watanabe (1993).

d) Increasing the strength of the external field, the value of the density contrast increases.

e) More asymmetrical initial configurations tend to have, at a given time, larger values of $\delta$. Then internal and external shear produce a more rapid evolution of the density contrast.

In order to study the effect of shear on the density contrast at turnaround and velocity at collapse, I re-derived the equations of the density contrast at turn-around and the velocity at collapse time of the BS model, taking account of both internal and external shear. The results have shown that:

(aa) The values of density contrast and collapse velocity at collapse time are always reduced with respect to the spherical collapse, in agreement with WS, BS, Szalay \& Silk (1983).

(bb) The effects of the slowing down of the collapse obtained in this paper (similar to that of Peebles 1990) are smaller than that we would find if we had used a system having internal substructure. 
(cc) The shear has a big impact on our view of structure formation:

(cc1) its first consequences are a change in the mass function, the two-point correlation function, and the mass that accretes on density peaks (see also Del Popolo \& Gambera 2000; Audit et al. 1997; Del Popolo \& Gambera 1999; Del Popolo et al. 1999; Peebles 1993).

(cc2) Another important consequence of the results described is connected to the value of the density parameter, $\Omega$. When using the spherical infall model we underestimate the value of the density parameter (Szalay \& Silk 1983; Lee et al. 1986; Taruya \& Soda 2000), since shear slows down the collapse infalling velocity.

Almost all the results obtained with the analytical model were tested against numerical solutions, always finding good agreement with them.

Acknowledgements. I would like to thank Prof. E. Recami, E. Nihal Ercan, A. Diaferio, J. D. Barrow and Y. Ekşi for some useful comments.

Finally, I would like to thank The Boğaziçi University Research Foundation for the financial support through the project code $01 B 304$.

\section{References}

Aarseth, S. J., \& Binney, J. 1978, MNRAS, 185, 227

Adams, M. T., Strom, K. M., \& Strom, S. E. 1980, ApJ, 238, 445

Antonuccio-Delogu, V., \& Colafrancesco, S. 1994, ApJ, 427, 72

Audit, E., \& Alimi, J. M. 1996, A\&A, 315, 11

Audit, E., Teyssier, R., \& Alimi, J. M. 1997, A\&A, 325, 439

Audit, E., Teyssier, R., \& Alimi, J. M. 1998, A\&A, 333, 779

Bardeen, J. M., Bond, J. R., Kaiser, N., \& Szalay, A. S. 1986, ApJ, 304, 15

Barnes, J., \& Efstathiou, G. 1987, ApJ, 319, 575

Barrow, J. D., \& Silk, J. 1981, ApJ, 250, 432

Bertschinger, E., \& Jain, B. 1994, ApJ, 431, 486

Binggeli, B. 1982, A\&A, 107, 338

Binney, J., \& Silk, J. 1979, MNRAS, 188, 273

Bond, J. R., \& Myers, S. T. 1993a, preprint CITA/93/27

Bond, J. R., \& Myers, S. T. 1993b, preprint CITA/93/28

Carter, D., \& Metcalfe, N. 1980, MNRAS, 191, 325

Catelan, P., \& Theuns, T. 1996a, MNRAS, 282, 436

Catelan, P., \& Theuns, T. 1996b, MNRAS, 282, 455

Chernin, A. D. 1970, Soviet Phys. JETP Lett., 11, 210

Davis, M., \& Peebles, P. J. E. 1977, ApJS, 34, 425

Del Popolo, A., \& Gambera, M. 1997, A\&A, 321, 691

Del Popolo, A., \& Gambera, M. 1998, A\&A, 337, 96

Del Popolo, A., \& Gambera, M. 1999, A\&A, 344, 17

Doroshkevich, A. G. 1970, Astrophysics, 3, 320

Dressler, A. 1981, ApJ, 243, 26

Eisenstein, D. J., \& Loeb, A. 1995, ApJ, 439, 520

Engineer, S., Kanekar, N., \& Padmanabhan, T. 2000, MNRAS, 314,279

Evrard, A. E., \& Crone, M. M. 1992, ApJ, 394, L1
Gonzales, A. 1995 [astro-ph/9506026]

Gregory, S. A., \& Tifft, W. G. 1976, ApJ, 205, 716

Heavens, A., \& Peacock, J. A. 1988, MNRAS, 232, 339

Hoffman, Y. 1986a, ApJ, 308, 493

Hoffman, Y. 1986b, ApJ, 301, 65

Hoffman, Y. 1989, ApJ, 340, 69

Hoyle, F. 1949, in IAU and International Union of Theorethicaland Applied Mechanics Symposium, 195

Hui, L., \& Bertschinger J. 1995 [astro-ph/9508114]

Lambas, D. G., Groth, E. J., \& Peebles, P. E. J. 1988, ApJ, 95, 996

Lambas, D. G., Nicotra, M., Muriel, H., \& Ruiz, L. 1990, AJ, 100,1006

Lee, H., Hoffman, Y., \& Ftaclas, C. 1986, ApJ, 304, L11

Lilje, P. B., Yahil, A., \& Jones, B. J. T. 1986, ApJ, 297, 27

Peebles, P. J. E. 1969, ApJ, 155, 393

Peebles, P. J. E., \& Groth, E. J. 1976, A\&A, 53,131

Peebles, P. J. E. 1980, The large scale structure of the universe (Princeton University Press)

Peebles, P. J. E. 1990, ApJ, 365, 27

Peebles, P. J. E. 1993, Principles of Physical Cosmology (Princeton University Press)

Pichon, C., \& Bernardeau, F. 1999, A\&A, 343, 663

Plionis, M., Valdarnini, R., \& Jing, Y. 1992, ApJ, 398, 12

Plionis, M. 1994, ApJS, 95, 401

Porciani, C., Dekel, a., \& Hoffman, Y. 2002 [astro-ph/0105165]

Rhee, G. F. R. N., \& Katgert, P. 1987, A\&A, 183, 217

Rood, H. H., Page, T. L., Kintner, E. C., \& King, I. R. 1972, ApJ, 175, 627

Ryden, B. S., \& Gunn, J. E. 1987, ApJ, 318, 15

Ryden, B. S. 1988, ApJ, 329, 589

Salvador-Solé, E., \& Solanes, J. M. 1993, ApJ, 417, 427

Sasaki, M., \& Kasai, M. 1998, Prog. Theor. Phys., 99, 585

Sheth, R. K., \& Jain, B. 1997, MNRAS, 285, 231

Szalay, A. S., \& Silk, J. 1983, ApJ, 264, L31

Taruya, A., \& Soda, J. 2000, MNRAS, 317, 873

Tucker, G. S., \& Peterson, J. B. 1988, AJ, 95, 298

Tully, R. B. 1982, ApJ, 257, 389

van Kampen, E., \& Rhee, G. F. R. N. 1990, A\&A, 237, 283

van de Weygaert, R., \& Babul, A. 1994, ApJ, 425, L59

van de Weygaert, R. 1996, Astronomical Society of the Pacific

Conf. Ser. 94, ed. P. Coles, V. Martinez, \& Maria-Jesus Pons-Borderia (see also [astro-ph/9601026]), 49

Villumsen, J. V., \& Davis, M. 1986, ApJ, 308. 499

Watanabe, T., \& Inagaki, S. 1991, PASJ, 43, 413

Watanabe, T., \& Inagaki, S. 1992, PASJ, 44,561

Watanabe, T. 1993, PASJ, 45, 393

West, M. J. 1989, ApJ, 347, 610

West, M. J. 1994, MNRAS, 268, 79

White, S. D. M., \& Silk, J. 1979, ApJ, 231, 1

Zel'dovich, Ya. B. 1970, Astr. Ap., 5, 84

Zeldovich, Ya. B. 1978, Proc. IAU Symp., 79, The Large Scale Structure of the Universe, ed. M. S. Longair, \& J. Einasto (Dordrecht), 409

Zel'dovich, Ya. B., \& Novikov 1983, Relativistic Astrophysics, vol. 2, The Structure and Evolution of the Universe (Chicago: University of Chicago Press) 